\title{
Activation of MrgprA3 and MrgprC11 on Bladder- Innervating Afferents Induces Peripheral and Central Hypersensitivity to Bladder Distension
}

\author{
Luke Grundy, ${ }^{1,2 *}$ Ashlee Caldwell, ${ }^{1,2 *}$ Sonia Garcia-Caraballo, ${ }^{1,2}$ David Grundy, ${ }^{3}{ }^{\circledR}$ Nick J. Spencer, ${ }^{4}$ \\ Xinzhong Dong, ${ }^{5}$ Joel Castro, ${ }^{1,2}$ Andrea M. Harrington, ${ }^{1,2}$ and ${ }^{\circledR}$ Stuart M. Brierley ${ }^{1,2,6}$ \\ ${ }^{1}$ Visceral Pain Research Group, College of Medicine and Public Health, Flinders Health and Medical Research Institute, Flinders University, Bedford Park, \\ 5042 South Australia, Australia, ${ }^{2}$ Hopwood Centre for Neurobiology, Lifelong Health Theme, South Australian Health and Medical Research Institute, North \\ Terrace, Adelaide, 5000 South Australia, Australia, ${ }^{3}$ Department of Biomedical Science, University of Sheffield, Sheffield, S10 2TN, United Kingdom, ${ }^{4}$ Visceral \\ Neurophysiology Laboratory, Flinders Health and Medical Research Institute, College of Medicine and Public Health, Flinders University, Adelaide, 5042 South \\ Australia, Australia, ${ }^{5}$ Solomon H. Snyder Department of Neuroscience, Center for Sensory Biology, School of Medicine, Howard Hughes Medical Institute, \\ Johns Hopkins University, Baltimore, 21205 Maryland, and ${ }^{6}$ Discipline of Medicine, University of Adelaide, Adelaide, 5000 South Australia, Australia
}

Understanding the sensory mechanisms innervating the bladder is paramount to developing efficacious treatments for chronic bladder hypersensitivity conditions. The contribution of Mas-gene-related G protein-coupled receptors (Mrgpr) to bladder signaling is currently unknown. Using male and female mice, we show with single-cell RT-PCR that subpopulations of DRG neurons innervating the mouse bladder express MrgprA3 (14\%) and MrgprC11 (38\%), either individually or in combination, with high levels of coexpression with Trpv1 (81\%-89\%). Calcium imaging studies demonstrated MrgprA3 and MrgprC11 agonists (chloroquine, BAM8-22, and neuropeptide FF) activated subpopulations of bladder-innervating DRG neurons, showing functional evidence of coexpression between MrgprA3, MrgprC11, and TRPV1. In ex vivo bladder-nerve preparations, chloroquine, BAM8-22, and neuropeptide FF all evoked mechanical hypersensitivity in subpopulations (20\%-41\%) of bladder afferents. These effects were absent in recordings from $\mathrm{Mrgpr}$-cluster $\Delta^{-/-}$ mice. In vitro whole-cell patch-clamp recordings showed that application of an MrgprA3/C11 agonist mixture induced neuronal hyperexcitability in $44 \%$ of bladder-innervating DRG neurons. Finally, in vivo instillation of an MrgprA3/C11 agonist mixture into the bladder of WT mice induced a significant activation of dorsal horn neurons within the lumbosacral spinal cord, as quantified by pERK immunoreactivity. This MrgprA3/C11 agonist-induced activation was particularly apparent within the superficial dorsal horn and the sacral parasympathetic nuclei of WT, but not $M r g p r-c l u s t e r \Delta^{-1-}$ mice. This study demonstrates, for the first time, functional expression of MrgprA3 and MrgprC11 in bladder afferents. Activation of these receptors triggers hypersensitivity to distension, a critically valuable factor for therapeutic target development.

Key words: bladder; GPCR; itch; pain; sensory neurons; visceral afferents

\section{Significance Statement}

Determining how bladder afferents become sensitized is the first step in finding effective treatments for common urological disorders such as overactive bladder and interstitial cystitis/bladder pain syndrome. Here we show that two of the key receptors, MrgprA3 and MrgprC11, that mediate itch from the skin are also expressed on afferents innervating the bladder. Activation of these receptors results in sensitization of bladder afferents, resulting in sensory signals being sent into the spinal cord that prematurely indicate bladder fullness. Targeting bladder afferents expressing MrgprA3 or MrgprC11 and preventing their sensitization may provide a novel approach for treating overactive bladder and interstitial cystitis/bladder pain syndrome.

Received Dec. 24, 2020; revised Feb. 28, 2021; accepted Mar. 6, 2021

Author contributions: L.G., A.C., and S.M.B. designed research; L.G., A.C., and S.G.-C. performed research; L.G., A.C., S.G.-C., J.C., A.M.H., and S.M.B. analyzed data; L.G., A.C., S.G.-C., D.G., N.J.S., X.D., J.C., A.M.H., and S.M.B. edited the paper; S.M.B. wrote the first draft of the paper; S.M.B. wrote the paper.

X.D. is a cofounder of Escient Pharmaceuticals. All remaining authors declare no competing financial interests.

This work was supported by National Health and Medical Research Council of Australia Project Grant APP1140297 to S.M.B., National Health and Medical Research Council of Australia R.D. Wright Biomedical
Research Fellowship APP1126378 to S.M.B., and Australian Research Council Discovery Project DP180101395 to A.M.H. and S.M.B.

* L.G. and A.C. contributed equally to this work.

Correspondence should be addressed to Stuart M. Brierley at stuart.brierley@flinders.edu.au.

https://doi.org/10.1523/JNEUROSCI.0033-21.2021

Copyright $\odot 2021$ the authors 


\section{Introduction}

Patients suffering from common urological disorders, such as overactive bladder and interstitial cystitis/bladder pain syndrome, experience chronic urinary urgency, frequency, and discomfort or pain (Abrams et al., 2002; Homma et al., 2020). Hypersensitivity of the peripheral afferents innervating the bladder are considered a key component in the underlying symptomology of these conditions (de Groat et al., 2015; Grundy et al., 2018a; de Groat and Yoshimura, 2009b). Numerous nociceptive ion channels and receptors, including TRPV1 and P2X3, have been identified as contributing to bladder afferent hypersensitivity, and have been subsequently explored to develop novel treatments for bladder disorders (Daly et al., 2007; de Groat and Yoshimura, 2009b; DeBerry et al., 2014; Yoshimura et al., 2014; Grundy et al., 2018c; Spencer et al., 2018; Andersson, 2019). However, other mechanisms are also likely involved. In this regard, cues can be taken from sensations that originate from the skin, whereby itch and pain are commonly experienced protective sensations that occur in response to irritant or noxious stimuli (Steinhoff et al., 2018). Both of these processes require activation and sensitization of sensory afferents that project from the skin into the dorsal horn of the spinal cord where they provide input to the CNS (T. Liu and Ji, 2013; LaMotte et al., 2014; Schmelz, 2015; J. S. Lee et al., 2016). Interestingly, itch can be mediated by histamine release from immune cells, which activate histamine receptors on sensory afferents (Shim and Oh, 2008), while several histamine-independent itch mechanisms have also been described. These include those dependent on Mas-related G-protein coupled receptors (Mrgprs) (Tominaga and Takamori, 2014; Zhu et al., 2017), a large subfamily of GPCRs predominantly expressed in the DRG (Dong et al., 2001; Lembo et al., 2002). In particular, MrgprA3 has been identified as the receptor responsible for itch associated with the antimalarial drug chloroquine (Q. Liu et al., 2009), while MrgprC11 activation via the endogenous peptide agonist bovine adrenal medulla 8-22 (BAM8-22) or mast cell mediator neuropeptide FF (NPFF), a dual MrgprC11 and A4 agonist (Q. Liu et al., 2009) also initiate pruritus and scratching (M. G. Lee et al., 2008; Sikand et al., 2011).

Traditionally these mechanisms have been considered unique to the skin; however, pruritogenic-like mechanisms are being increasingly identified in the viscera. The same histamine receptors that mediate pruritus have been explored in a number of visceral organs, including the gastrointestinal tract and bladder, where histamine has been found to activate and induce hypersensitivity of visceral afferents (Kreis et al., 1998; Wouters et al., 2016; Grundy et al., 2020). More recently, MrgprC11 and MrgprA3 have been localized in subpopulations of colon-innervating afferents (Castro et al., 2019; Van Remoortel et al., 2019), as well as vagal afferents innervating the lungs (Han et al., 2018). Activation of MrgprA3 and MrgprC11 expressed on colonic afferents results in colonic hypersensitivity, enhanced abdominal pain, and altered animal behaviors (Castro et al., 2019; Van Remoortel et al., 2019), effects that were significantly increased in an animal model of chronic visceral hypersensitivity (Castro et al., 2019). Given the relative similarities in the extrinsic sensory afferent innervation of the colon and bladder, including the ability to encode a variety of nociceptive and non-nociceptive stimuli (Brierley et al., 2004; Zagorodnyuk et al., 2006; Xu and Gebhart, 2008; Grundy et al., 2019a), we sought to explore the expression of key Mrgprs in the bladder and their role in mediating neuronal excitability and bladder sensory signaling.

Here, we show that MrgprC11, MrgprA3, and to a much smaller extent MrgprD are expressed in subpopulations of bladder-innervating DRG neurons. Agonists of MrgprC11 and MrgprA3 cause pronounced afferent hypersensitivity to bladder distension ex vivo, activate and evoke hyperexcitability of bladder-innervating DRG neurons in vitro, and enhance activation of neurons within the dorsal horn of the spinal cord in vivo.

\section{Materials and Methods}

\section{Ethics and animals}

In this study, a total of 12 - to 18 -week-old male $(N=39)$ and female $(N=21) \mathrm{C} 57 \mathrm{BL} / 6 \mathrm{~J}$ mice from an in-house breeding colony at the South Australian Health and Medical Research Institute (SAHMRI) were used. Mice were acquired from an in-house C57BL/6J breeding program (JAX strain \#000664; originally purchased from The Jackson Laboratory, breeding barn MP14) and then bred within the specific and opportunistic pathogen-free animal care facility at SAHMRI. A genetically modified mouse strain lacking a cluster of MRGPR genes (Mrgpr-cluster $\left.\Delta^{-1-}\right)$, including MrgprA3 and MrgprC11, was utilized for some experiments (Q. Liu et al., 2009). The Mrgpr-cluster $\Delta^{-1-}$ mice were a generous gift from Prof. Xinzhong Dong (Howard Hughes Medical Institute, Johns Hopkins University) and then bred in-house at SAHMRI as per the C57BL/6 J mice. All experiments were approved by and performed in accordance with the approval of the SAHMRI Animal Ethics Committee (SAM195 and SAM190). Mice were group housed (up to 5 mice per cage) within individual ventilated cages filled with chip coarse dust-free aspen bedding (catalog \#ASPJMAEBCA; PuraBed), with free access to LabDiet JL Rat and Mouse/Auto6F chow (catalog \#5K52) and autoclaved reverse osmosis water. Cages were stored within a temperature-controlled environment of $22^{\circ} \mathrm{C}$ and a $12 \mathrm{~h}$ light/ $12 \mathrm{~h}$ dark cycle on individual ventilated cage racks. Experiment sample sizes are detailed in the corresponding figure legends.

\section{Ex vivo bladder afferent nerve recordings}

Nerve recording was performed using a previously described ex vivo model in male mice ( $N=5 /$ cohort) (Grundy et al., 2018b,c, 2019b, 2020; Konthapakdee et al., 2019). Mice were humanely killed via $\mathrm{CO}_{2}$ inhalation, and the entire lower abdomen was removed and submerged in a modified organ bath under continual perfusion with gassed $\left(95 \% \mathrm{O}_{2}\right.$ and $5 \% \mathrm{CO}_{2}$ ) Krebs-bicarbonate solution (composition in mM as follows: $118.4 \mathrm{NaCl}, 24.9 \mathrm{NaHCO}_{3}, 1.9 \mathrm{CaCl}_{2}, 1.2 \mathrm{MgSO}_{4}, 4.7 \mathrm{KCl}, 1.2 \mathrm{KH}_{2} \mathrm{PO}_{4}$, 11.7 glucose) at $35^{\circ} \mathrm{C}$. The bladder, urethra, and ureters were exposed by removing excess tissue. Ureters were tied with 4-0 perma-hand silk (Ethicon, \#LA53G). The bladder was catheterized (PE 50 tubing) through the urethra and connected to a syringe pump (NE-1000) to allow a controlled fill rate of $30 \mu \mathrm{l} / \mathrm{min}$ with saline ( $\mathrm{NaCl}, 0.9 \%)$. A second catheter was inserted through the dome of the bladder, secured with silk, and connected to a pressure transducer (NL108T2; Digitimer) to enable intravesical pressure recording during graded distension. Pelvic nerves, isolated from all other nerve fibers between the pelvic ganglia and the spinal cord, were dissected into fine multiunit branches; and a single branch was placed within a sealed glass pipette containing a microelectrode (WPI) attached to a Neurolog headstage (NL100AK; Digitimer). Nerve activity was amplified (NL104), filtered (NL 125/126, bandpass 50-5000 Hz, Neurolog; Digitimer), and digitized (CED 1401; Cambridge Electronic Design) to a PC for offline analysis using Spike2 software (Cambridge Electronic Design). The number of action potentials crossing a preset threshold at twice the background electrical noise was determined per second to quantify the afferent response. Single-unit analysis was performed offline by matching individual spike waveforms through linear interpolation using Spike2 version 5.18 software. A single unit was deemed to be responsive to an agonist if a $>20 \%$ increase in neuronal excitability (impulses/s across all distension pressures $0-30$ $\mathrm{mmHg}$ area under the curve) was detected.

Afferent recording experimental protocols. At the start of each afferent recording experiment, control bladder distensions were performed with intravesical infusion of saline $(\mathrm{NaCl}, 0.9 \%)$ at a rate of $30 \mu \mathrm{l} / \mathrm{min}$ to a maximum pressure of $30 \mathrm{mmHg}$ at $10 \mathrm{~min}$ intervals to assess the viability of the preparation and reproducibility of the intravesical pressure and neuronal responses to distension. The volume in the bladder was 
extrapolated from the known fill rate $(30 \mu \mathrm{l} / \mathrm{min})$ and the time taken (s) to reach maximum pressure $(30 \mathrm{mmHg})$. Compliance was determined by plotting intravesical pressure against the calculated volume. After a stable baseline was maintained, the saline in the infusion pump was replaced by BAM8-22 (20 $\mu \mathrm{M}$; Tocris Bioscience; \#1763), chloroquine (100 $\mu \mathrm{M}$; Sigma Millipore, \#PHR1258-1G), NPFF (50 $\mu \mathrm{M}$; Tocris Bioscience; \#3137), or an "itch cocktail" containing all three agonists combined (BAM8-22: $20 \mu \mathrm{M}$, chloroquine: $100 \mu \mathrm{M}$, and NPFF: $50 \mu \mathrm{M}$ ). All experimental compounds were dissolved in $\mathrm{dH}_{2} \mathrm{O}$ to form stock solutions, which were frozen at $-80^{\circ} \mathrm{C}$ and defrosted immediately before being used for each experiment. The single nerve bundle isolated and inserted into the glass electrode during the dissection process was contained within the recording electrode for the entire experiment, allowing multiunit afferent nerve recordings to be performed and comparisons between afferent firing rates to be compared in the same nerve fibers during intrabladder incubation with saline and Mrgpr agonists.

\section{Retrograde tracing from the bladder}

A small, aseptic, abdominal incision was made in anesthetized (2\%-4\% isoflurane in oxygen) male $(N=9)$ and female $(N=3)$ mice. A $5 \mu \mathrm{l}$ Hamilton syringe attached to a 30 -gauge needle was used to inject cholera toxin subunit B conjugated to AlexaFluor-488 (CTB-488, $0.5 \%$ diluted in $0.1 \mathrm{~m}$ PBS, pH 7.4; Thermo Fisher Scientific) at three sites into the bladder wall ( $3 \mu \mathrm{l} /$ injection) (Grundy et al., 2018a,b, 2020). To prevent injection of CTB into the bladder lumen, the needle was inserted subserosally, parallel with the bladder muscle. The abdominal incision was then sutured closed and analgesic (buprenorphine [Temvet]; $0.1 \mathrm{mg} / \mathrm{kg}$; Troy Laboratories, APVMA \#67612) and antibiotic (amoxicillin; $50 \mathrm{mg} / \mathrm{kg}$; Amoxil, AUSTR11137) administered subcutaneously as mice regained consciousness. Following laparotomy, mice were individually housed and allowed to recover. After $4 \mathrm{~d}$, mice were humanely killed by $\mathrm{CO}_{2}$ asphyxiation and the lumbosacral (LS; L5-S1) DRG removed for subsequent isolation and culture of the neurons to visualize CTB-labeled bladder innervating neurons among the DRG neurons.

\section{$m R N A$ expression analysis in $D R G$, isolated urothelial cells, and bladder} mucosal and detrusor layers

Tissue collection and urothelial cell isolation. Sixteen- to 18-week-old male $(N=6)$ and female $(N=8)$ mice were humanely killed via $\mathrm{CO}_{2}$ inhalation and the bladder and lumbosacral (L5-S1) DRG removed. DRGs were frozen in dry ice in pairs by spinal level (L5, L6, S1) and stored at $-80^{\circ} \mathrm{C}$ for RNA extraction and qRT-PCR. The bladder was opened, stretched out and pinned flat, urothelial side up. For tissue qRT-PCR, the mucosal and detrusor layers were gently peeled apart, frozen in dry ice, and stored at $-80^{\circ} \mathrm{C}$ for RNA extraction and qRT-PCR. For urothelial cell qRT-PCR, urothelial cells were isolated as performed previously (Grundy et al., 2018c, 2020; Spencer et al., 2018). Following a rinse in sterile PBS, the bladder was incubated for $3 \mathrm{~h}$ at room temperature in DMEM containing $2.5 \mathrm{mg} / \mathrm{ml}$ dispase (Invitrogen, Thermo Fisher Scientific, \#17105041), 10\% FCS, and $1 \mu \mathrm{m} / \mathrm{ml}$ HEPES (Sigma Millipore, \#H3375, pH 7.0). A blunt scalpel was used to collect cells by gentle scraping of the urothelium. Cells were dissociated in $0.025 \%$ trypsin EDTA (Invitrogen, Thermo Fisher Scientific, \#25200072) at $37^{\circ} \mathrm{C}$ in $5 \% \mathrm{CO}_{2}$ for $10 \mathrm{~min}$ with gentle intermittent trituration. The cell suspension was then added to DMEM containing 10\% FCS to deactivate the trypsin before centrifugation $\left(15 \mathrm{~min}, 1500 \mathrm{rpm}, 4^{\circ} \mathrm{C}\right)$. Following aspiration of supernatant, cells were resuspended in keratinocyte serum-free media (Invitrogen, \#17005042), and cell count and viability were determined using the Countess Automated Cell Counter (Invitrogen, Thermo Fisher Scientific). Cells were pelleted, frozen in dry ice, and stored at $-80^{\circ} \mathrm{C}$ for RNA extraction and qRT-PCR.

RNA extraction. RNA was extracted using the PureLink RNA Micro kit (Invitrogen, \#12183-016; DRG pairs and isolated urothelial cells) or the PureLink RNA Mini kit (Invitrogen, \#12183018A; bladder mucosa and detrusor) with DNase treatment (Invitrogen, \#12185-010) according to the manufacturer's instructions. A NanoDrop Lite spectrophotometer (Thermo Fisher Scientific) was used to determine RNA purity and quantity. Using SuperScript VILO Master Mix (Invitrogen, \#11755250), RNA
Table 1. Primers used for $q R T-P C R$ and RT-PCR receptor expression assays

\begin{tabular}{lll}
\hline Gene alias & Gene target & Assay ID \\
\hline$\beta$-actin (reference gene) & Actb & Mm00607939_s1 \\
HPRT (reference gene) & Hprt & Mm00446968_m1 \\
Gapdh (reference gene) & Gapdh & Mm99999915_g1 \\
Tubulin-3 (neuronal marker) & Tubb3 & Mm00727586_s1 \\
Mas-related G-protein receptor A3 & MrgprA3 & Mm02620679_s1 \\
Mas-related G-protein receptor C11 & MrgprX1 & Mm02525847_g1 \\
Mas-related G-protein receptor D & MrgprD & Mm01701850_s1 \\
Transient receptor potential vanilloid 1 & Trpv1 & Mm01246300_m1 \\
Transient receptor potential ankyrin 1 & Trpa1 & Mm01227437_m1 \\
Transient receptor potential vanilloid 4 & Trpv4 & Mm00499025_m1 \\
\hline
\end{tabular}

was reverse-transcribed to $\mathrm{CDNA}$ as per the manufacturer's instructions. cDNA was then stored at $-20^{\circ} \mathrm{C}$ for qRT-PCR.

$q R T-P C R$. qRT-PCR was performed using TaqMan Gene Expression Master Mix (Applied Biosystems, \#4369016) with commercially available hydrolysis probes (TaqMan; Invitrogen; for details, see Table 1) and RNase-free water (Ambion, \#AM9916). For each reaction, $10 \mu \mathrm{l}$ of qPCR MasterMix, $1 \mu \mathrm{l}$ of TaqMan primer assay, $4 \mu \mathrm{l}$ of water, and $5 \mu \mathrm{l}$ of cDNA (1:2 dilution in RNA-free $\mathrm{H}_{2} \mathrm{O}$ ) from each sample were tested in duplicate for each target. Endogenous controls Actb ( $\beta$-actin; DRG pairs) and Gapdh and Hprt (glyceraldehyde 3-phosphate dehydrogenase and hypoxanthine phosphoribosyltransferase 1; mucosal and detrusor tissue layers and isolated urothelial cells) were used as endogenous controls. Assays were run for 45 cycles on a 7500 Fast Real-Time PCR System (Applied Biosystems) machine, using 7500 Fast software, version 2.0.6. mRNA quantities are expressed as $2^{-\Delta \mathrm{Ct}}$ relative to reference gene Actb (DRG pairs) or Gapdh $x$ Hprt (geometric mean; mucosa, detrusor, and urothelial cells) (Grundy et al., 2018b,d; Castro et al., 2019).

\section{Cell culture of bladder-innervating DRG neurons}

Four days following retrograde tracing, mice were humanely killed via $\mathrm{CO}_{2}$ inhalation and lumbosacral (LS; L5-S1) DRGs were removed. DRGs were digested in HBSS ( $\mathrm{pH} 7.4$, Invitrogen, \#14170161) containing $2.5 \mathrm{mg} / \mathrm{ml}$ collagenase II (Invitrogen, Thermo Fisher Scientific, \#17101015) and $4.5 \mathrm{mg} / \mathrm{ml}$ dispase (Invitrogen, Thermo Fisher Scientific, \#17105041) at $37^{\circ} \mathrm{C}$ for $30 \mathrm{~min}$. Following aspiration of the collagenase-dispase solution, DRGs were incubated in HBSS containing collagenase $(4.5 \mathrm{mg} / \mathrm{ml})$ only for $10 \mathrm{~min}$ at $37^{\circ} \mathrm{C}$. After subsequent washes in HBSS, trituration through firepolished Pasteur pipettes of descending diameter in $600 \mu \mathrm{l}$ complete DMEM (Invitrogen, Thermo Fisher Scientific, \#11995065), 10\% FCS (Invitrogen, Thermo Fisher Scientific), $2 \mathrm{~mm}$ L-glutamine (Invitrogen, Thermo Fisher Scientific, \#25030081), $100 \mu \mathrm{M}$ MEM nonessential amino acids (Invitrogen, Thermo Fisher Scientific, \#11140076), $100 \mathrm{mg} / \mathrm{ml}$ penicillin/streptomycin (Invitrogen, Thermo Fisher Scientific, \#15070063), and $96 \mu \mathrm{g} / \mathrm{L}$ nerve growth factor-7S (Sigma Millipore, N0513-0.1MG) mechanically disrupted DRGs and dissociated cells, which were then centrifuged for $1 \mathrm{~min}$ at $50 \times g$ (Osteen et al., 2016; Inserra et al., 2017; Grundy et al., 2018a,b). Supernatant was gently aspirated, and neurons resuspended in $360 \mu \mathrm{l}$ complete DMEM and spot-plated $(30 \mu \mathrm{l})$ onto laminin $(20 \mu \mathrm{g} / \mathrm{ml}$; Sigma Millipore, \#L2020) and poly-D-lysine $(800 \mu \mathrm{g} / \mathrm{ml}$; Thermo Fisher Scientific) coated $13 \mathrm{~mm}$ coverslips. Coverslips were incubated for 2-3 h at $37^{\circ} \mathrm{C}$ in $5 \% \mathrm{CO}_{2}$ to allow adherence of neurons before flooding with $1.7 \mathrm{ml}$ complete DMEM. Cultured neurons were then maintained in an incubator at $37^{\circ} \mathrm{C}$ in $5 \% \mathrm{CO}_{2}$ for $18-48 \mathrm{~h}$ for calcium imaging, patch-clamp recordings, or $4 \mathrm{~h}$ for cell picking for single-cell RT-PCR (Grundy et al., 2018b; Castro et al., 2019).

\section{Single-cell RT-PCR of individual bladder-innervating DRG neurons}

Under continuous perfusion of sterile and RNA-/DNase-free PBS, single retrogradely traced bladder DRG neurons were identified using a fluorescence microscope and collected into the end of a fine glass capillary using a micromanipulator (Grundy et al., 2018b). The glass capillary containing the cell was then broken into a sterile Eppendorf tube containing $10 \mu \mathrm{l}$ of lysis buffer with DNase (TaqMan Gene Expression Cellsto-CT Kit; Invitrogen, \#4399002). A bath control was also taken for each coverslip of cells and analyzed concurrently with cells. After lysis and 
DNase treatment, samples were immediately frozen on dry ice and stored at $-80^{\circ} \mathrm{C}$ until cDNA synthesis was performed. RNA was reverse-transcribed to cDNA using SuperScript IV VILO Master Mix with ezDNase Enzyme (Invitrogen, \#11766500) as per the manufacturer's instructions. cDNA was then stored at $-20^{\circ} \mathrm{C}$ for real-time PCR. Tubulin- 3 expression was used as a neuronal marker and positive control. Expression of each target gene within a single cell was determined by looking at the $\log (\Delta \mathrm{Rn})$ curve against cycle number. Only amplification curves that exhibited an exponential phase that rose steadily and eventually plateaued within 50 cycles were counted.

Calcium imaging of cultured DRG neurons

Cultured DRG neurons (18-48 h) were loaded with $2.5 \mu \mathrm{M}$ fura-2-acetoxymethyl ester (fura-2 AM; Invitrogen, Thermo Fisher Scientific, \#F1221) in 0.01\% pluronic F-127 (Invitrogen, Thermo Fisher Scientific, $\#$ P3000MP) at $37^{\circ} \mathrm{C}$ for $30 \mathrm{~min}$ followed by a $10 \mathrm{~min}$ wash with HEPES buffer (10 mm HEPES sodium salt, Sigma Millipore, \#H7006-100G), 140 $\mathrm{mm} \mathrm{NaCl}$ (Chem Supply, \#SA046-3KG), $4 \mathrm{~mm} \mathrm{KCl} \mathrm{(Chem} \mathrm{Supply,}$ \#PA054-500G), 5 mm D-glucose anhydrous (Chem Supply, \#GA018500G), $2 \mathrm{~mm} \mathrm{CaCl}_{2}$ (Scharlau, \#CA01951000), and $2 \mathrm{~mm} \mathrm{MgCl}_{2}$ (Sigma Millipore, \#M8266-100G), pH 7.40, before imaging at room temperature $\left(23^{\circ} \mathrm{C}\right)$ (Bellono et al., 2017; Grundy et al., 2018b, 2020). Emissions for fura-2 AM were measured at $510 \mathrm{~nm}$, following excitation at 340 and $380 \mathrm{~nm}$, using a Nikon TE300 Eclipse microscope equipped with a Sutter DG-4/OF wavelength switcher, an Omega XF04 filter set for fura-2 AM, a Photonic Science ISIS-3 intensified CCD camera, and Universal Interface Card MetaFluor software. Retrogradely traced bladder DRG neurons were identified by the presence of the CTB-488, visible with excitation at $480 \mathrm{~nm}$. Excitation at $480 \mathrm{~nm}$ was used only as an initial bladder neuron identifier; to prevent unnecessary photobleaching, images were not obtained at $480 \mathrm{~nm}$ throughout the experiment. Fluorescence images were obtained every 2 s using a $20 \times$ objective. Data were recorded and further analyzed using MetaFluor software.

After an initial baseline reading to ensure cell fluorescence was stable, indicating healthy cells, DRG neurons were stimulated with chloroquine (1 $\mu \mathrm{M}$; Sigma Millipore, \#PHR1258-1G), BAM8-22 (2 $\mu \mathrm{M}$; Tocris Bioscience, \#1763), and NPFF (5 $\mu$; Tocris Bioscience, \#3137) with changes in intracellular calcium $\left(\mathrm{Ca}^{2+}{ }_{\mathrm{i}}\right)$ monitored in real time. The TRPV1 receptor agonist capsaicin ( $3 \mu \mathrm{M}$; Sigma Millipore, M2028) was applied at the end of experiments. $\mathrm{Ca}^{2+}{ }_{\mathrm{i}}$ is expressed as the ratio between the fluorescence signals at 340 and $380 \mathrm{nM}$ (fura-2 AM, 340/ 380).

\section{Patch-clamp electrophysiology of bladder-innervating DRG neurons}

Current-clamp recordings. Whole-cell patch-clamp recordings in current-clamp mode were made from retrogradely traced fluorescently labeled lumbosacral (L5-S1) bladder-innervating DRG neurons using borosilicate glass pipettes (WPI) fire-polished to 3-7 $\mathrm{m} \Omega$ and back-filled with an intracellular solution consisting of the following (in $\mathrm{mM}$ ): 135 $\mathrm{KCl}, 2 \mathrm{MgCl}_{2}, 2 \mathrm{MgATP}, 5$ EGTANa, 10 HEPES-Na, adjusted to $\mathrm{pH} 7.3$, $275 \mathrm{mOsm}$. Rheobase (amount of current required to fire an action potential) was determined in normal extracellular bath solution (in $\mathrm{mm}$ as follows: $140 \mathrm{NaCl}, 4 \mathrm{KCl}, 2 \mathrm{MgCl}_{2}, 2 \mathrm{CaCl}_{2}, 10 \mathrm{HEPES}, 5$ glucose, adjusted to $\mathrm{pH} 7.4,285 \mathrm{mOsm}$ ) by application of a series of depolarizing pulses from a holding potential of $-65 \mathrm{mV}$ ( 10 or $25 \mathrm{pA}$ increments [ $480 \mathrm{~ms}$ ]) followed by hold at $-65 \mathrm{mV}(100 \mathrm{~ms})$. Cells were continually perfused with bath solution by a gravity-driven multibarrel perfusion system positioned within $1 \mathrm{~mm}$ of the neuron under investigation (Osteen et al., 2016; Grundy et al., 2018b,d). After baseline recordings in external bath solution, an Mrgpr agonist itch cocktail (MrgprA3 agonist; chloroquine $10 \mu \mathrm{M}$; Sigma Millipore, \#PHR1258-1G; MrgprC11 agonist; BAM8-22: $2 \mu \mathrm{M}$, Tocris Bioscience, \#1763; MrgprC11/A4 agonist; NPFF $5 \mu \mathrm{M}$; Tocris Bioscience, \#3137) was applied for a 2 min incubation period and a second recording was made.

In vivo spinal dorsal horn activation

In vivo bladder infusion. In vivo bladder infusion in anesthetized mice was performed as previously described (Grundy et al., 2018b, 2020). Twelve-week-old female mice $(N=5 /$ cohort) were anesthetized (isoflurane $2 \%-4 \%$ in oxygen), and a catheter (AS point style, $51 \mathrm{~mm}$, metal hub, 21-gauge needle [Hamilton, 7751-12]) was inserted into the bladder via the urethra. Correct catheter placement was determined by inserting gently until meeting resistance (bladder dome) and receding slightly $(2-3 \mathrm{~mm})$ to avoid damaging the bladder wall. Urine was removed using a suction syringe. Subsequently, a new catheter (AS point style, $51 \mathrm{~mm}$, metal hub, 21-gauge needle) was inserted, primed with either vehicle (saline) or an Mrgpr agonist mixture (BAM8-22: $20 \mu \mathrm{M}$; NPFF: $50 \mu \mathrm{M}$; and chloroquine: $100 \mu \mathrm{M}$ ), and $100 \mu \mathrm{l}$ of solution was then gently infused, without fully distending the bladder, and allowed to incubate for $5 \mathrm{~min}$. Immediately following removal of the compound, mice were administered an anesthetic overdose (intraperitoneal injection of pentobarbitone, $1.1 \mathrm{mg} / \mathrm{g}$ body weight; Lethabarb, Virbac Australia) followed by $4 \%$ buffered PFA transcardial perfuse fixation.

Transcardial perfuse fixation and tissue dissection. After anesthetic overdose, the thoracic cavity was opened and $0.5 \mathrm{ml}$ heparinized saline ( $50 \mathrm{IU}$ in $5 \mathrm{ml}$; Pfizer) was injected into the left ventricle. A 22-gauge needle attached to tubing and a peristaltic perfusion pump were then inserted into the injection site. The right atrium was cut open, allowing for perfusate drainage. Warm PB $\left(0.1 \mathrm{M} ; 21.7 \mathrm{~mm} \mathrm{NaH}_{2} \mathrm{PO}_{4}\right.$ [Chem Supply, \#SA061-500G], $81 \mathrm{~mm} \mathrm{Na}_{2} \mathrm{HPO}_{4}$ [VWR Chemicals, \#102494C], $\mathrm{pH}$ 7.2) was perfused, followed by ice-cold $4 \%$ PFA (Sigma Millipore, \#158127) in $0.1 \mathrm{M}$ PB. Following complete PFA perfusion, the lumbosacral spinal cord (determined by the level of DRG root insertion points; the lowest rib was used as an anatomic marker of DRG level T13) was removed and postfixed in $4 \% \mathrm{PFA}$ in $0.1 \mathrm{M} \mathrm{PB}$ at $4^{\circ} \mathrm{C}$ for $18-20 \mathrm{~h}$. After postfixation, spinal cord segments were cryoprotected in $30 \%$ sucrose/ PB (Sigma Millipore, \#S9378) for $48 \mathrm{~h}$ at $4^{\circ} \mathrm{C}$, followed by a $48 \mathrm{~h}$ incubation at $4^{\circ} \mathrm{C}$ in $50 \%$ optimal cutting temperature (VWR, \#C361603E)/ $30 \%$ sucrose/PB solution before freezing in $100 \%$ optimal cutting temperature using liquid nitrogen-cooled 2-methylbutane (Sigma Millipore, \#M32631). Frozen spinal cord segments were cryosectioned $(10 \mu \mathrm{m}$ thick) and placed onto gelatin-coated slides for immunostaining. Sections were performed serially and distributed over 6 slides, which were used for immunohistochemical localization of phosphorylated-MAP-kinase ERK 1/2 (pERK) (Grundy et al., 2018b, 2019b, 2020; Harrington et al., 2019). The number of pERK-immunoreactive ( $p E R K-I R)$ neurons were counted in L6-S2 spinal cord sections, with spinal segments determined ex vivo by the shape of the dorsal horn (Lein et al., 2007).

Immunohistochemistry of $p E R K$ within the spinal cord. The dorsal horn neurons activated by bladder infusion were identified by labeling for neuronal activation marker, pERK, using the DAKO Omnis autostainer (Agilent Technologies Australia), with DAB/HRP secondary antibody staining. Sections were processed via the University of Adelaide Health and Medical Sciences Histology Services. pERK activation was determined rather than c-Fos because of the rapidity of ERK phosphorylation (minutes compared with hours) (Gao and Ji, 2009). Given the short-duration nature of the specific peripheral stimuli applied, the rapid onset of pERK provides a more appropriate correlate. Further, quantification of pERK activation is increasingly being utilized to identify spinal activation to a range of peripheral stimuli (Jiang et al., 2015; Castro et al., 2017; Grundy et al., 2018a,b, 2020). After air drying for $1 \mathrm{~h}$, sections were postfixed in formalin with a brief rinse in distilled water $\left(\mathrm{dH}_{2} \mathrm{O}\right)$ before target retrieval at $97^{\circ} \mathrm{C}$ for 30 min with EnVision FLEX TRS Low $\mathrm{pH}$ target retrieval solution (citrate buffer, $\mathrm{pH}$ 6.1; K8005, Agilent DAKO), followed by a wash step with wash buffer (GC807, DAKO Omnis, Agilent). A 10 min protein block step with Serum-Free Protein Block (X0909, Agilent DAKO) to prevent nonspecific binding was followed by a $1 \mathrm{~h}$ incubation in primary antibody (pERK; 1:800) in Antibody Diluent (S0809, Agilent DAKO). Following removal of excess primary antibody with wash buffer, a 3 min incubation in Envision FLEX Peroxidase-blocking Reagent (GV823, Agilent DAKO) was performed. Slides were then washed twice with wash buffer before a $20 \mathrm{~min}$ incubation with Envision FLEX HRP Polymer (GV823, Agilent DAKO) for HRP binding. A further 2 wash steps were performed, followed by a DI rinse and a third wash step, before a 10 min incubation in EnVision FLEX Substrate Working Solution (DAB) for staining. Slides underwent alternate cycles of wash buffer, DI rinse, and wash buffer before a 3 min 
A

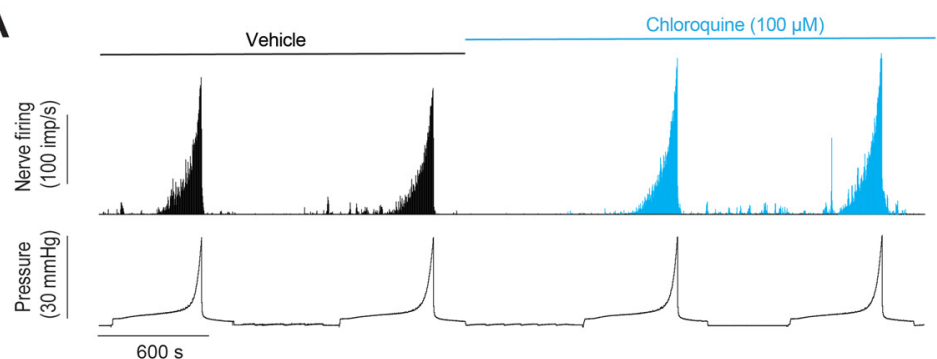

B

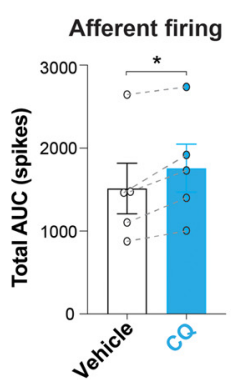

C

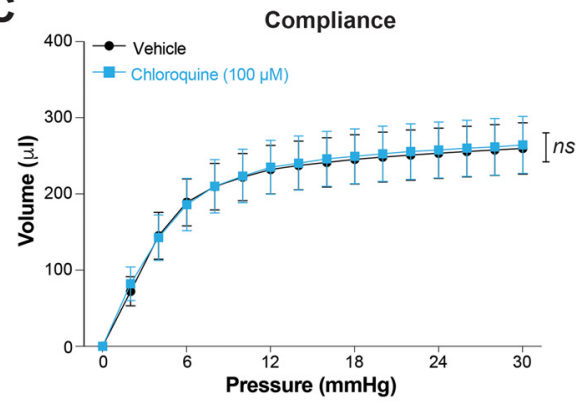

i)

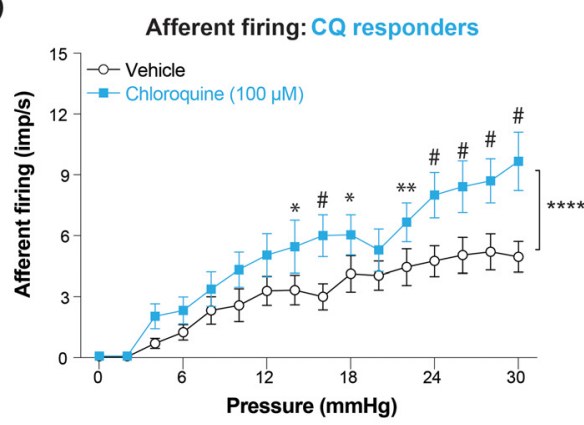

ii)

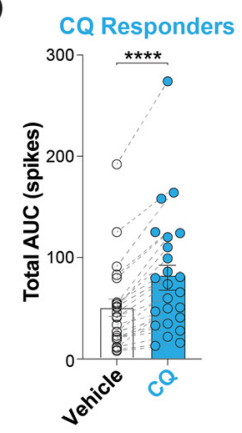

$E_{\text {i) }}$

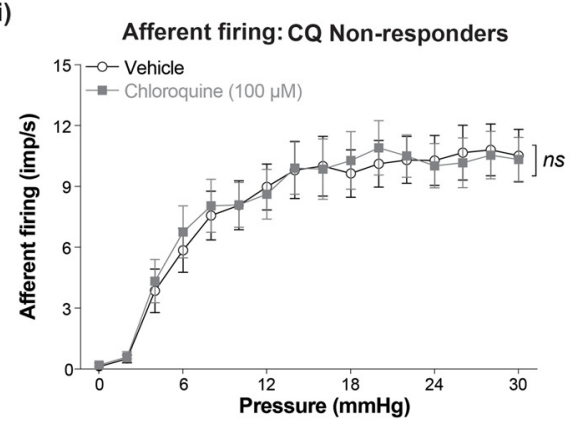

$\mathbf{F}$

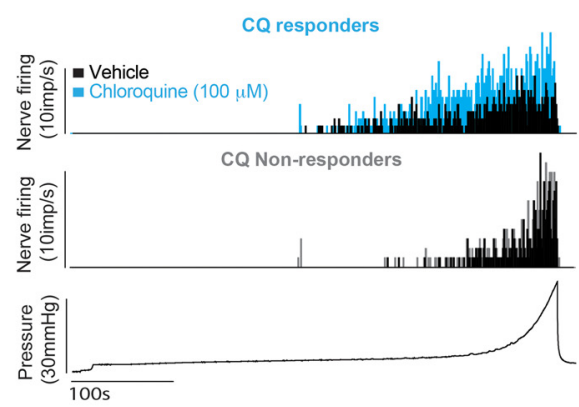

ii)

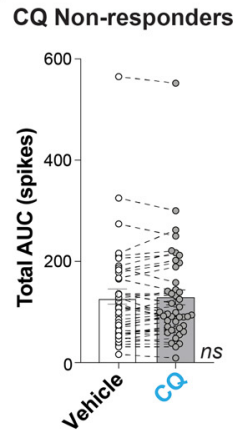

G

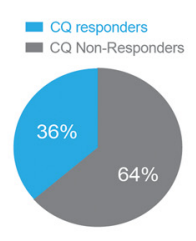

Figure 1. The MRGPRA3 agonist chloroquine evokes mechanical hypersensitivity in a cohort of bladder afferents. $A$, Original recordings from an ex vivo nerve recording preparation showing bladder afferent firing and intravesical pressure. Recordings show exaggerated whole nerve bladder afferent responses to distension following intravesical infusion with chloroquine $(100 \mu \mathrm{m}) . \boldsymbol{B}$, The sum total of afferent firing (total area under the curve; $0-30 \mathrm{mmHg}$ ) for each individual experiment showing bladder instillation with chloroquine $(100 \mu \mathrm{M})$ significantly increased bladder afferent firing to distension ( ${ }^{*} p<0.05$, paired $t$ test, $N=5$ mice). Dots indicate whole nerve responses from individual mice before and after the itch cocktail. $C$, The relationship between intrabladder

incubation with hematoxylin ready-to-use solution (K8018, Agilent DAKO) and a further DI rinse and wash step. Slides were removed from the DAKO Omnis, dehydrated in alcohol, and cleared in xylene before mounting with dibutylphthalate polystyrene xylene (Sigma Millipore) and coverslipping.

Microscopy. DAB/HRP-stained slides were imaged using a NanoZoomer microscope (2.0-HT, Hamamatsu), with 1-4 manually set focus points per section. Images of individual sections from L6 to S2 regions were taken using NanoZoomer digital pathology viewing software (NDP.view2, Hamamatsu) and analyzed using ImageJ software. The images were not manipulated in any way.

Spinal cord PERK neuronal counts and analysis. Neuronal counts were analyzed from previously saved digital photomicrographs, with only neurons with intact nuclei counted. The number of pERK-IR neurons in a quadrant of the L6-S2 dorsal horn was obtained from minimum 6 sections/animal viewed at $10 \times$ magnification. The mean number of pERK-IR neurons $( \pm$ SEM) in the superficial dorsal horn (SDH; LI-II), the dorsal gray commissure (DGC), and the sacral parasympathetic nuclei (SPN) in sacral spinal segments were compared between vehicle- and Mrgpr agonist mixturetreated mice. These regions are known to have roles in nociceptive signaling and autonomic reflexes (Todd, 2010) and are known to be activated by bladder afferent output (Grundy et al., 2018a, 2019a).

Experimental design and statistical analyses

Data are presented as mean \pm SEM or the percentage of afferents or neurons. Within the specific figure legends, $N$ indicates the number of animals, while $n$ indicates the number of independent afferents or neurons. Sample size was based on historical data and the use of power calculations. Statistical significance was reported at levels of $\left.p<0.05\left(^{*}\right), p<0.01\left(^{* *}\right), p<0.001{ }^{* * *}\right)$, and

$\leftarrow$

pressure and volume (compliance) during graded bladder distension $(30 \mu \mathrm{l} / \mathrm{min}$ from 0 to $30 \mathrm{mmHg})$ is unaffected by intravesical administration of chloroquine (100 $\mu \mathrm{m}$, not significant, $p>0.05$, repeated-measures two-way ANOVA, $N=5$ ). $\mathbf{D i}$, Post hoc singleunit analysis of the multiunit ex vivo bladder recordings shown in $\boldsymbol{A}$ identified bladder afferents that were responsive to chloroquine (showing a $>20 \%$ increase in afferent firing) in the presence of chloroquine $\left({ }^{* * *} p<0.0001\right.$, two-way repeated-measures ANOVA, $n=24$ afferents, $N=5$ mice). Significant differences were observed at the following distension pressures: $14 \mathrm{mmHg}(p=0.0127), 16 \mathrm{mmHg}$ $(p<0.0001), 18 \mathrm{mmHg}(p=0.0385), 22 \mathrm{mmHg}(p=0.0079), 24 \mathrm{mmHg}$ $(p<0.0001), 26 \mathrm{mmHg}(p<0.0001), 28 \mathrm{mmHg}(p<0.0001)$, and $30 \mathrm{mmHg} \quad(p<0.0001) \quad$ (Sidak's multiple comparisons tests, with ${ }^{*} p<0.05$, ${ }^{* *} p<0.01,{ }^{\#} p<0.001$ indicated on the graph). Dii, Chloroquine-responsive bladder afferents showing enhanced firing as determined by the total number of action potentials fired $\left({ }^{* * * *} p<0.0001\right.$, Wilcoxon matched-pairs signed rank test, $n=24$ afferents, $N=5$ mice). $\boldsymbol{E}$, Post hoc single-unit analysis also identified bladder afferents unaffected by chloroquine when analyzed (Ei) across the entire distension range (not significant, $p>0.05$, two-way repeated-measures ANOVA, $n=42$ afferents, $N=5$ mice) or (Eii) as the total number of action potentials fired (not significant, $p>0.05$, Wilcoxon matched-pairs signed rank test, $n=42$ afferents, $N=5$ mice). $\boldsymbol{F}$, Original recordings of chloroquine $(100 \mu \mathrm{m})$ responding (top) and nonresponding (middle) bladder afferents during controlled bladder distension from 0 to $30 \mathrm{mmHg}$ (bottom). G, Overall, $36 \%$ of single units (24 of 66) bladder afferents showed a $>20 \%$ increase in afferent firing to chloroquine. Data are mean \pm SEM. CQ, Chloroquine; AUC, area under the curve. Impulses per second: imp $/ \mathrm{s}^{-1}$. 
A

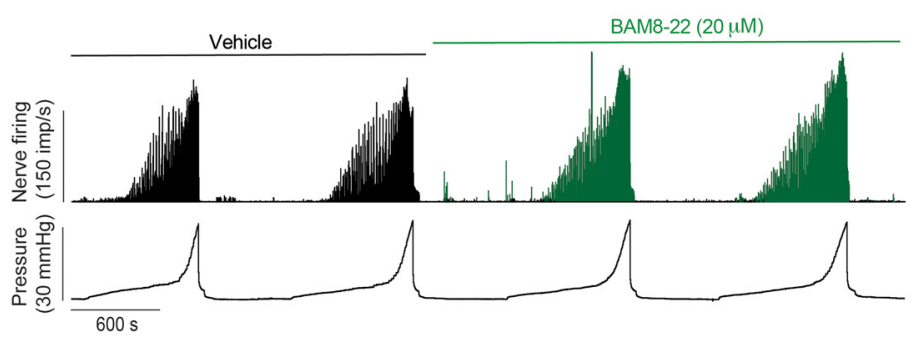

B

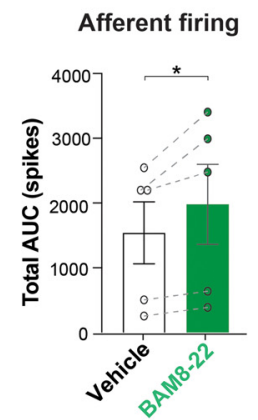

C

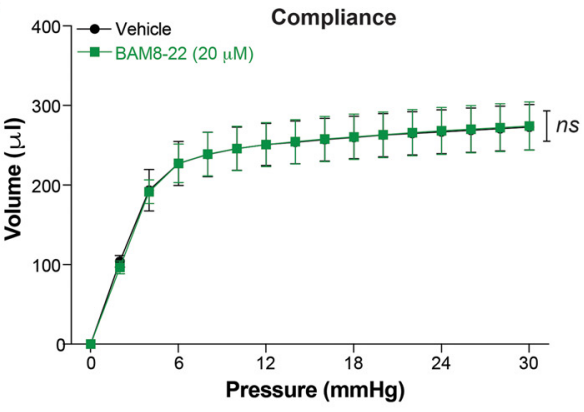

D

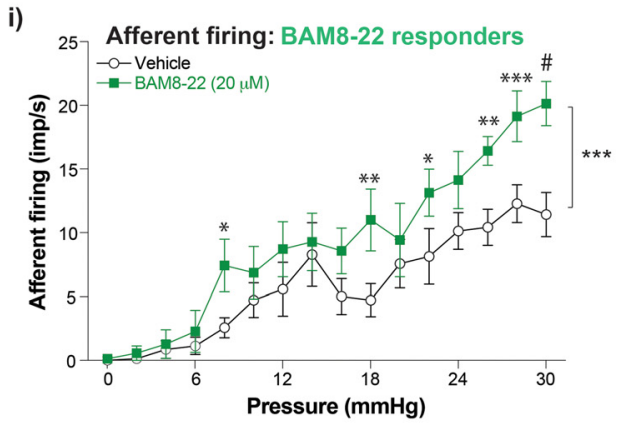

E

i) Afferent firing: BAM8-22 Non-responders

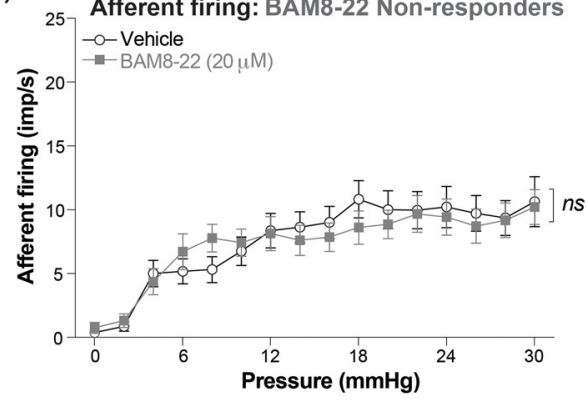

$\mathbf{F}$
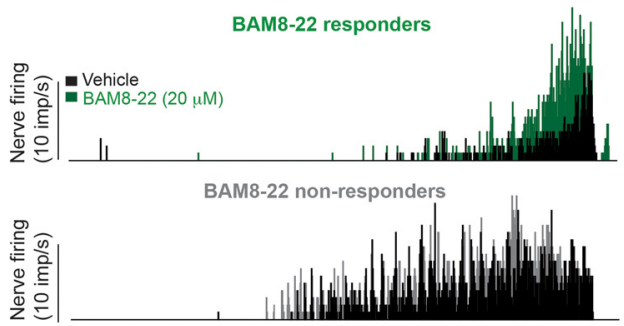

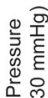

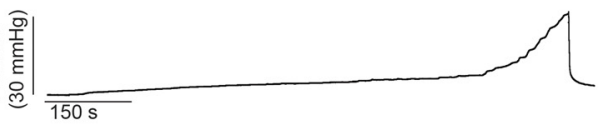

G

-BAM8-22 Responders -BAM8-22 Non-Responders

BAM8-22 Non-responders

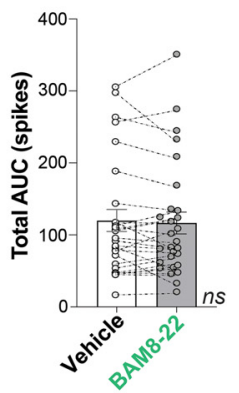

BAM8-22 responders
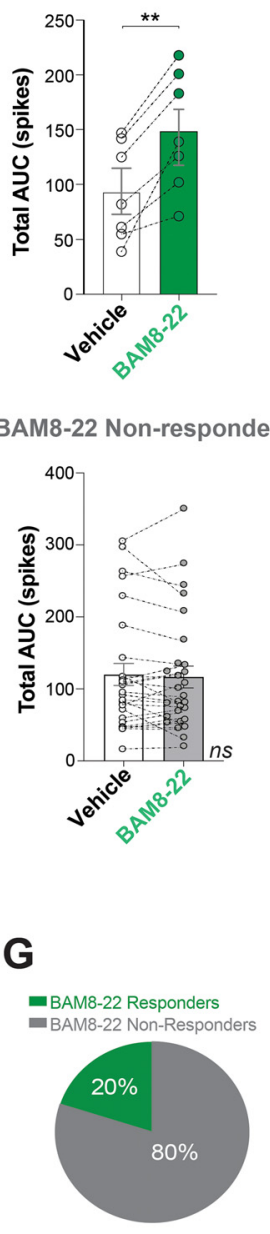

Figure 2. The MRGPRC11 agonist BAM8-22 evokes mechanical hypersensitivity in a cohort of bladder afferents. $A$, Original ex vivo recordings showing exaggerated bladder afferent responses to distension following intravesical infusion with BAM8-22 (20 $\mu \mathrm{M})$. B , Total bladder afferent firing was significantly increased following bladder instillation with BAM8-22 (20 $\mu \mathrm{m},{ }^{*} p<0.05$, paired $t$ test, $N=5$ mice). C, Bladder compliance during graded bladder distension is unaffected by intravesical administration of BAM8-22 (20 $\mu \mathrm{m}$, not significant, $p>0.05$, repeated-

$\left.p<0.0001^{* * *}\right)$. In some cases, because of space limitation, \# is used to indicate significance of $p<$ 0.0001 for multiple comparisons. Data were tested for Gaussian distribution using Prism 8 (GraphPad) to determine whether they were normally distributed or not and therefore the correct statistical tests to be used. Data were then analyzed accordingly using Prism 9.02 (GraphPad), via one- or two-way ANOVA with Tukey's or Sidak's post hoc analyses dependent on data distribution, or Student's $t$ tests, for parametric data. Nonparametric data were analyzed using KruskalWallis test with Dunn's multiple comparisons, MannWhitney analysis, or Wilcoxon matched-pairs signed rank test. The specific tests used for the analysis of each dataset, the $T, F$, and $p$ values, plus the number of $N$ or $n$ values are indicated within Results or within the individual figure legends.

\section{Results}

Mrgpr agonists induce distension evoked mechanical hypersensitivity of bladder afferents

In order to determine whether pruritogenic receptors have a functional role in bladder sensory signaling, we performed ex vivo bladder afferent recordings and determined their mechanosensitivity to bladder distension before and during bladder instillation with Mrgpr agonists. Intravesical application of the MrgprA3 agonist, chloroquine, evoked bladder afferent hypersensitivity to bladder distension in whole nerve recordings as determined by the total area under the curve of the response (Fig. $1 A, B ; t_{(4)}=3.816$, $p=0.0188$, paired $t$ test). Analysis of the pressure/volume relationship in the bladder during repetitive graded bladder distensions before and during chloroquine revealed no effect on bladder compliance (Fig. 1C; $F_{(15,64)}=0.3544, p=0.9856$, repeated-measures two-way ANOVA). This indicates that the mechanical hypersensitivity induced by bladder infusion of chloroquine is

$\leftarrow$

measures two-way ANOVA, $N=5$ ). $\boldsymbol{D}$, Post hoc single-unit analysis demonstrated a population of BAM8-22-responsive bladder afferents showing enhanced firing as determined (Di) across the distension range $\left({ }^{* *} p<0.001\right.$, two-way repeated-measures ANOVA, $n=7$ afferents, $N=5$ mice). Significant differences were observed at the following distension pressures: $8 \mathrm{mmHg} \quad(p=0.0331), 18 \mathrm{mmHg}$ $(p=0.0014), 22 \mathrm{mmHg} \quad(p=0.0248), 26 \mathrm{mmHg} \quad(p=0.0028)$, $28 \mathrm{mmHg}(p=0.0004)$, and $30 \mathrm{mmHg}(p<0.0001)$ (Sidak's multiple comparisons tests, with ${ }^{*} p<0.05,{ }^{* *} p<0.01$, ${ }^{* * *} p<0.001$, ${ }^{\#} p<0.0001$ indicated on the graph) or as (Dii) as the total number of action potentials fired $\left({ }^{* *} p<0.01\right.$, paired $t$ test, $n=7$ afferents, $N=5$ mice). $\boldsymbol{E}$, Post hoc single-unit analysis also identified bladder afferents unaffected by BAM8-22 when analyzed (EI) across the entire distension range (not significant, $p>0.05$, two-way repeated-measures ANOVA, $n=28$ afferents, $N=5$ mice) or as (Eii) the total number of action potentials fired (not significant, $p>0.05$, Wilcoxon matched-pairs signed rank test, $n=28$ afferents, $N=5$ mice). $\boldsymbol{F}$, Original recordings of BAM8-22 responding and nonresponding bladder afferents during bladder distension from 0 to $30 \mathrm{mmHg}$. G, Overall, $20 \%$ (7 of 35) of bladder afferents showed a $>20 \%$ increase in afferent firing to BAM8-22. Data are mean \pm SEM. Impulses per second: $\mathrm{imp} / \mathrm{s}^{-1}$. 
A

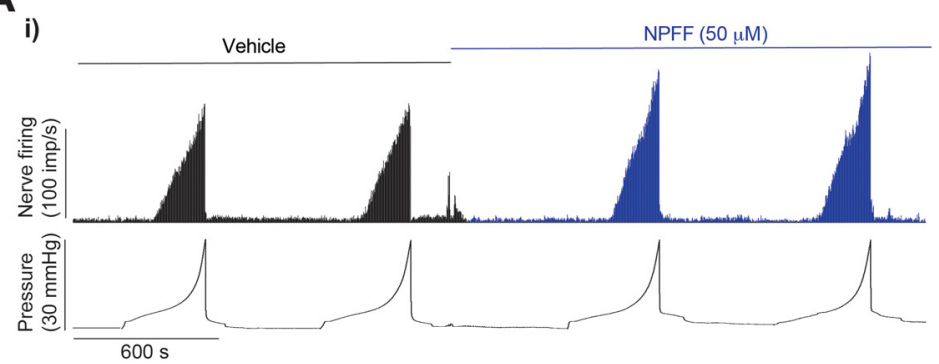

B

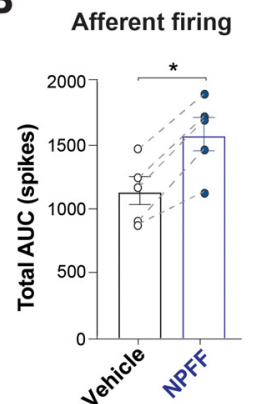

C

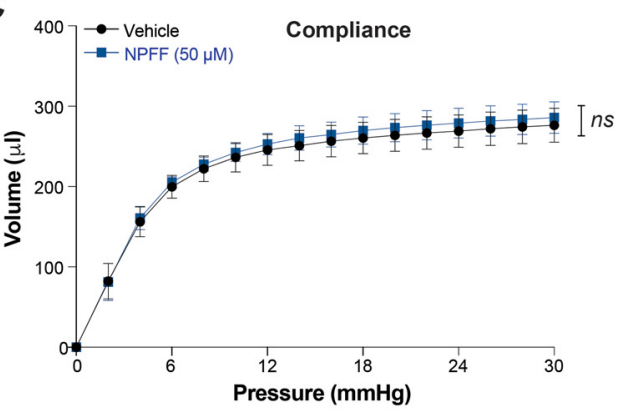

Di)

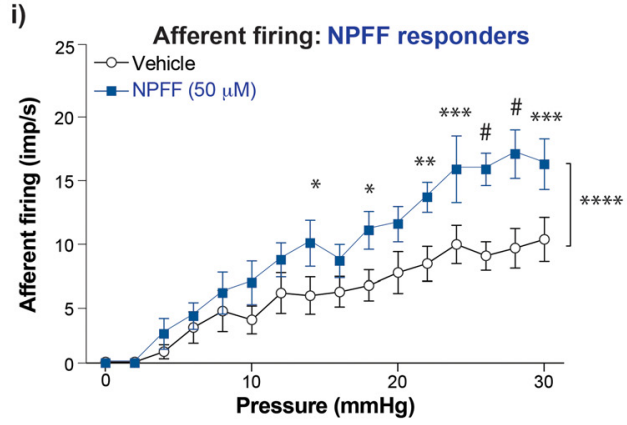

ii)

E

i) Afferent firing: NPFF Non-responders

ii)

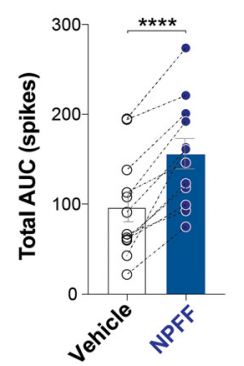

ii)

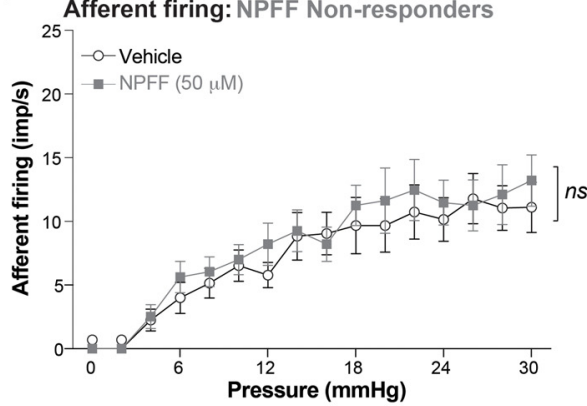

$\mathbf{F}$

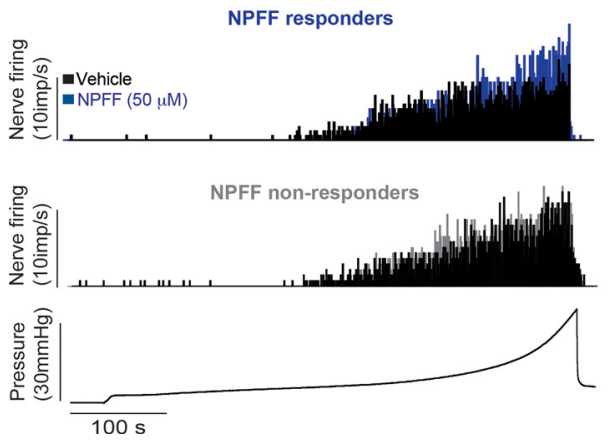

\section{G}

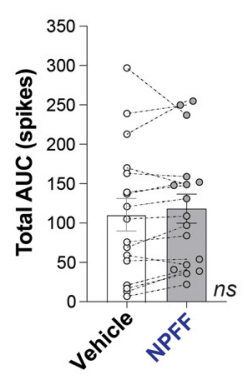

not secondary to changes in bladder muscle function, which can indirectly influence bladder afferent responsiveness. To further investigate the afferent response, we performed post hoc single-unit analysis. This analysis revealed that distinct populations of bladder afferents became hypersensitive following chloroquine application (called responders) when analyzed across all distension pressures (Fig. $1 D i ; F_{(15,368)}=4.163, p<0.0001$, repeated-measures two-way ANOVA) or measuring the total afferent response (Fig. 1Dii; $p<0.0001$, baseline: mean $49.17 \pm$ SEM 8.595 vs chloroquine; $81.54 \pm 12.15$, Wilcoxon matched-pairs). In contrast, other afferents were unaffected by chloroquine application (called nonresponders) when measured across all distension pressures (Fig. $1 E i ; F_{(15,656)}=0.4762$, $p=0.9526$, repeated-measures two-way ANOVA) or as the total afferent response (Fig. 1Eii; $p=$ 0.4365, baseline: $127.1 \pm 14.97$ vs CQ: $129.2 \pm 14.69$, Wilcoxon matched-pairs). Of the afferents that were affected by chloroquine, the predominant effects were apparent throughout most distension pressures from 14 to $30 \mathrm{mmHg}$ (for full details, see Fig. $1 D i, F$; Sidak's multiple comparisons test, $p=0.0385-p<0.0001$ ). Overall, $36 \%$ of bladder afferents were responsive to chloroquine application (Fig. 1G).

To follow-up on these findings, we also used the MrgprC11 agonist BAM8-22 and found that it also significantly enhanced bladder afferent responses to bladder distension (Fig. $2 A, B ; t_{(4)}=2.797, p=0.0490$, paired $t$ test), without affecting muscle compliance (Fig. 2C; $F_{(15,48)}=0.1091, p>0.9999$, repeated-measures two-way ANOVA). Single-unit analysis of bladder afferents revealed that BAM8-22 also sensitized a subpopulation of bladder afferents (Fig. $2 D i, F_{(15,96)}=2.921, p=0.0008$, repeated-measures two-way ANOVA; Fig. 2Dii, $t_{(6)}=5.596, p=$ 0.0014 , paired $t$ test). Responsive afferents were particularly affected at distension pressures $\geq 18$ $\mathrm{mmHg}$ (for full details, see Fig. 2Di,F; Sidak's multiple comparisons test, $p=0.0331-p<0.0001$ ). In

\section{$\leftarrow$}

administration of NPFF ( $5 \mu \mathrm{m}$, not significant, $p>0.05$, repeated-measures two-way ANOVA, $N=5$ for each group). $\boldsymbol{D}$, Post hoc single-unit analysis showed a population of NPFF-responsive bladder afferents showing enhanced firing (Di) across distension pressures $\left({ }^{* * *} p<0.0001\right.$, twoway repeated-measures ANOVA, $n=12$ afferents, $N=5$ mice). Significant differences were observed at the following distension pressures: $14 \mathrm{mmHg}$ $(p=0.028), 18 \mathrm{mmHg}(p=0.017), 22 \mathrm{mmHg}(p=0.0014), 24 \mathrm{mmHg}$ $(p=0.0002), 26 \mathrm{mmHg}(p<0.0001), 28 \mathrm{mmHg}(p<0.0001)$, and $30 \mathrm{mmHg} \quad(p=0.0002) \quad$ (Sidak's multiple comparisons tests, with ${ }^{*} p<0.05,{ }^{* *} p<0.01,{ }^{* * *} p<0.001,{ }^{\#} p<0.0001$ indicated on the graph) or, as in Dii, the total number of action potentials fired ( ${ }^{* * *} p<0.0001$, paired $t$ test, $n=12$ afferents, $N=5$ mice). $E$, A population of bladder afferents were unresponsive to NPFF based on (Ei) responses across the distension range (not significant, $p>0.05$, two-way repeated-measures ANOVA, $n=17$ afferents, $N=5$ ) or (Eii) the total number of action potentials fired (not significant, $p>0.05$, paired $t$ test, $n=17$ afferents, $N=5$ mice). $\boldsymbol{F}$, Example traces of responding and nonresponding bladder afferents to NPFF $(50 \mu \mathrm{M})$ during bladder distension. G, Overall, $41 \%$ of single unit (12 of 29) bladder afferents showed a $>20 \%$ increase in afferent firing to NPFF. Data are mean \pm SEM. Impulses per second: imp/s $\mathrm{s}^{-1}$.
Figure 3. The MRGPRC11/A4 agonist NPFF evokes mechanical hypersensitivity in a cohort of bladder afferents. $\boldsymbol{A i}$, Ex vivo recordings show an enhanced afferent response to distension following intravesical infusion with NPFF $(50 \mu \mathrm{m})$. B, Total bladder afferent firing was significantly increased following bladder instillation with NPFF (50 $\mu \mathrm{m},{ }^{*} p<0.05$, paired $t$ test, $N=5$ mice). C, Bladder compliance is unaffected by intravesical 


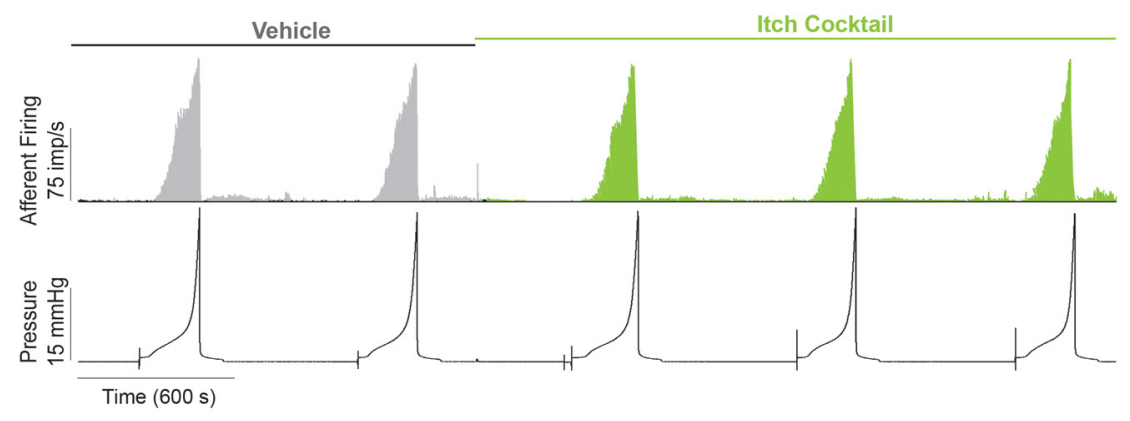

B

i)

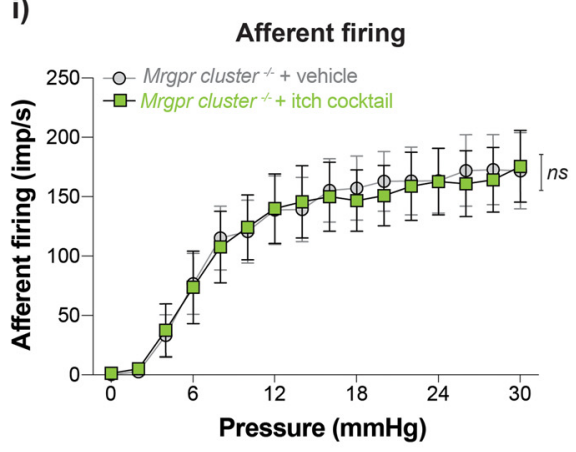

ii)

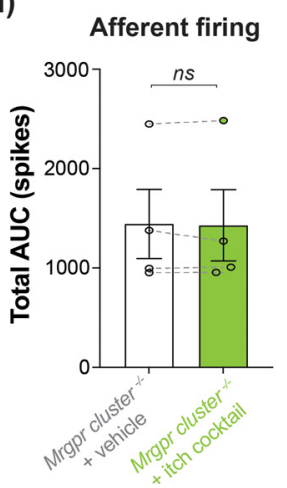

C

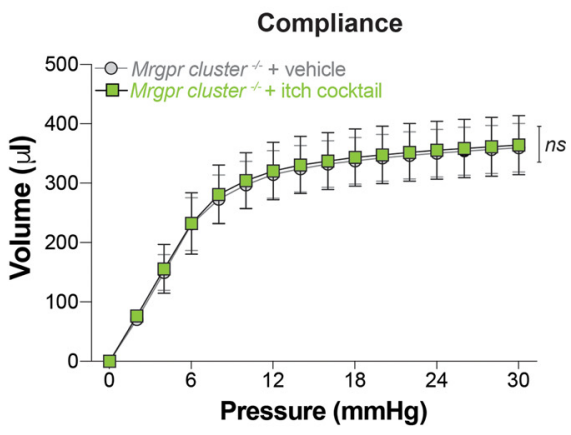

Figure 4. Bladder afferent responses to an itch cocktail are absent in Mrgpr-cluster $\Delta^{-1-}$ mice. A, Original recordings of bladder afferent firing and intravesical pressure during consecutive bladder distensions (0-30 mmHg) with saline followed by the itch cocktail containing the Mrgpr agonists chloroquine $(100 \mu \mathrm{m})$, BAM8-22 (20 $\mu \mathrm{m})$, and NPFF (50 $\mu \mathrm{m})$. Afferent responses were stable during saline infusion and unchanged after administration of the Mrgpr agonists. Bi, Group data from ex vivo afferent recordings using Mrgpr-cluster $\Delta^{-1-}$ mice showing almost identical responses to bladder instillation of vehicle (saline) or an itch cocktail (not significant, $p>0.05$, two-way repeated-measures ANOVA, $N=4$ mice). Bii, Total bladder afferent firing was unchanged following bladder instillation of an itch cocktail (not significant, $p>0.05$, paired $t$ test, $N=4$ mice). Dots indicate whole nerve responses from individual mice before and after the itch cocktail. C, Bladder compliance during graded bladder distension $(30 \mu \mathrm{l} / \mathrm{min}, 0-30 \mathrm{mmHg})$ was unaffected by intravesical administration of the Mrgpr agonist itch cocktail in Mrgprcluster $\Delta^{-1-}$ mice (not significant, $p>0.05$, two-way repeated-measures ANOVA, $N=4$ ). Data are mean \pm SEM.

contrast, another population was unaffected by BAM8-22 Fig. $2 E i, F_{(15,864)}=0.4244, p=0.9724$, repeated-measures two-way ANOVA; and Fig. 2Eii, $p=0.6832$, baseline: $120.2 \pm 15.38$ vs BAM8-22: 116.6 \pm 15.09 , Wilcoxon matched pairs). Responsive afferents constituted $20 \%$ of the bladder afferents recorded (Fig. $2 G)$.

We also found that the MrgprC11/MrgprA4 agonist NPFF evoked significant bladder afferent hypersensitivity (Fig. $3 A, B$; $t_{(4)}=8.091, p=0.0013$, paired $t$ test) that was independent of changes in muscle compliance (Fig. $3 C ; F_{(15,48)}=0.2828$, $p=0.995$, repeated-measures two-way ANOVA). NPFF affected the responsiveness of $41 \%$ of bladder afferents recorded (Fig. $3 D i, F_{(15,144)}=3.417, p<0.001$, repeated-measures two-way ANOVA, Fig. 3Dii, $t_{(11)}=7.526, p<0.0001$, paired $t$ test). In contrast, NPFF did not affect another population of bladder afferents (Fig. $3 E i, F_{(15,576)}=0.1821, p=0.9998$, repeated-measures two-way ANOVA; Fig. 3Eii: $t_{(16)}=1.194, p=0.250$, paired $t$ test; Fig. 3F,G). Neither chloroquine, BAM8-22, nor NPFF recruited "silent" afferents to become subsequently mechanosensitive. Overall, these findings show that chloroquine, BAM8-22, and NPFF all evoke hypersensitivity to distension in subpopulations of bladder afferents, suggesting the Mrgpr receptors they activate are expressed on specific subpopulations of bladderinnervating DRG neurons.

In order to confirm that these effects were mediated via Mrgpr activation, we performed additional ex vivo bladder afferent recordings with tissue from $M r g p r-c l u s t e r \Delta^{-1-}$ mice (Fig.
$4 A$ ). In our previous studies focusing on colonic sensitivity, we have shown that a combined application of Mrgpr agonists, as an itch cocktail, causes pronounced colonic mechanical hypersensitivity, an effect that is absent in Mrgpr-cluster $\Delta^{-1-}$ mice (Castro et al., 2019). In the current study, we found that ex vivo bladder afferent responses to graded distension in Mrgpr-cluster $\Delta^{-1-}$ mice were identical when comparing between the application of vehicle or the itch cocktail of coapplied chloroquine, BAM8-22, and NPFF (Fig. $4 B i, F_{(15,48)}=0.606, p=0.854$, repeated-measures two-way ANOVA, Fig. 4 Bii: $t_{(3)}=0.463, p=0.675$, paired $t$ test). Additionally, these studies also showed that bladder compliance was unaltered in vehicle or itch cocktail treated bladders from Mrgpr-cluster $\Delta^{-1-}$ mice (Fig. $4 C, F_{(15,48)}=0.046, p>0.999$, repeated-measures two-way ANOVA). These findings confirm the role of Mrgprs in chloroquine, BAM8-22, and NPFF induced bladder afferent mechanical hypersensitivity.

\section{MrgprA3 and MrgprC11 are expressed in subpopulations of bladder-innervating DRG neurons}

To determine the mechanisms by which Mrgpr agonists induce distension-evoked bladder afferent hypersensitivity, we determined Mrgpr expression in DRG, as well as in bladder tissue. Lumbosacral DRGs contain the cell bodies of sensory neurons innervating a number of different pelvic organs, including $>30 \%$ of which innervate the mouse bladder (Christianson et al., 2007; Grundy et al., 2018d). Therefore, we performed retrograde tracing from the bladder wall to isolate those that innervate the 
A

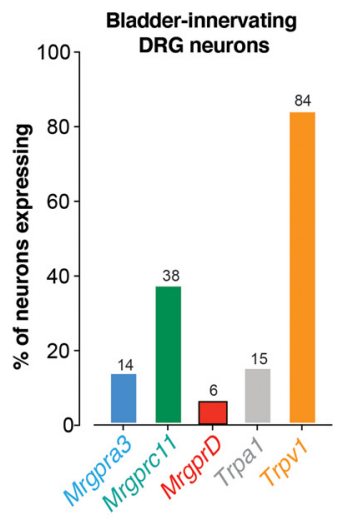

C

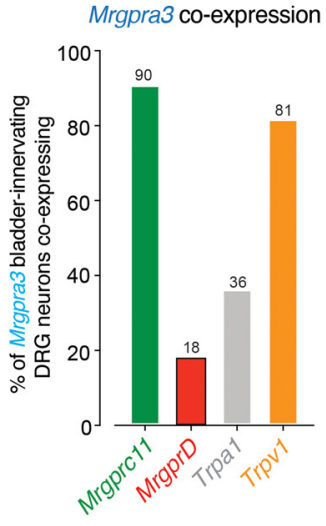

B

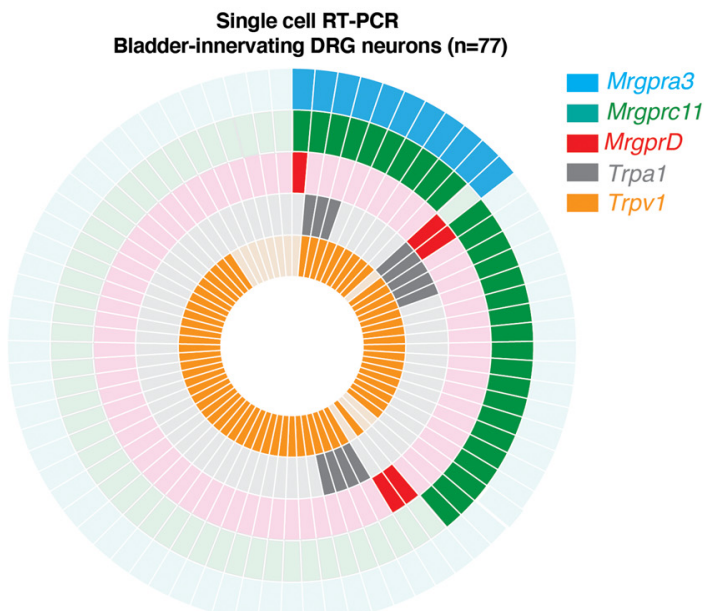

D

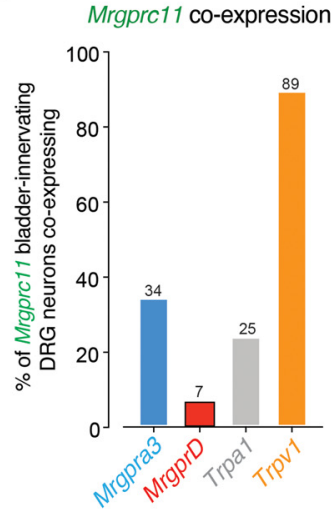

E

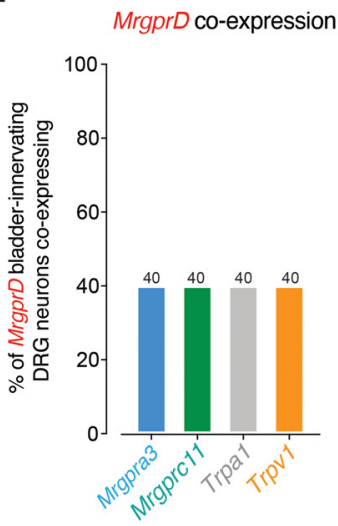

F

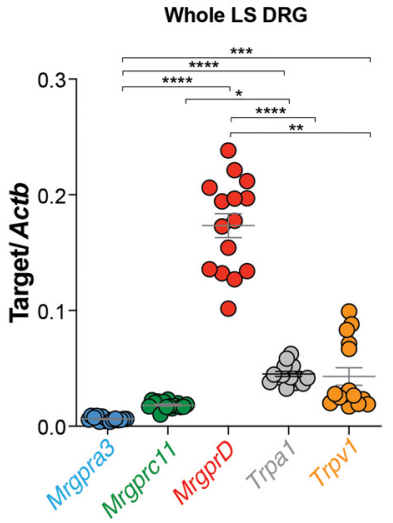

G

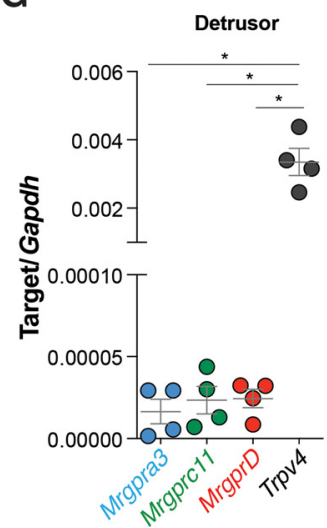

H

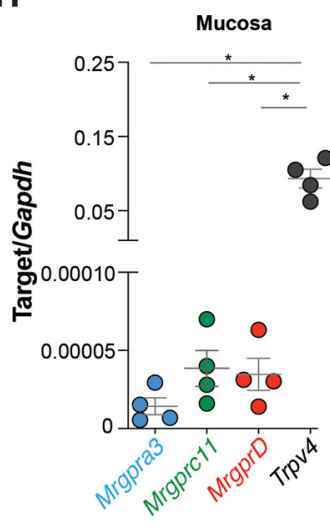

I

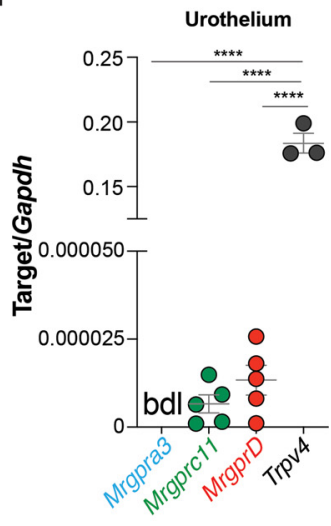

Figure 5. Mrgpr receptors are expressed by bladder-innervating DRG neurons. $A$, Single-cell RT-PCR of retrogradely traced bladder-innervating DRG neurons reveals the percentage of neurons expressing and coexpressing genes encoding Mrgprs and Trp channels ( $n=77$ single cells from $N=6$ mice). mRNA for MrgprA3 and MrgprC 11 was expressed in $14 \%$ and $38 \%$ of bladderinnervating DRG neurons, respectively. In contrast, MrgprD was expressed in only $6 \%$ of bladder-innervating DRG neurons. Trpa1 and Trpv1 mRNA transcripts were detected in $15 \%$ and $84 \%$ of bladder-innervating DRG neurons. B, Donut plot showing expression and coexpression of genes encoding MrgprC11, MrgprA3, MrgprD, Trpa1, and Trpv1 in 77 individual retrogradely traced bladder-innervating DRG neurons. Each color represents an individual gene. Bold coloring represents expression. MrgprA3 is represented in the outer ring, with Trpv1 in the inner ring. Individual neurons are arranged radially, such that coexpression of genes in a single neuron can be easily identified running from outside to inside. $C$, The majority of bladder-innervating DRG neurons that express MrgprA3 coexpress MrgprC11 (90\%) and Trpv1 (81\%). In contrast, a minor proportion of MrgprA3-expressing bladder DRG neurons also coexpress MrgprD (18\%) or Trpa1 (36\%). D, MrgprC11 is commonly coexpressed with $\operatorname{Trpv} 1$ (89\%), but less so with MrgprA3 (34\%), MrgprD (7\%), and Trpa1 (25\%). E, Of the very few cells that express MrgprD (5 of 77 neurons), 40\% (2 of 5 neurons) coexpress with MrgprA3, MrgprC11, Trpv1, or Trpa1. F, mRNA expression of MrgprA3, MrgprC11, MrgprD, Trpv1, and Trpa1 in whole LS (L5-S1) DRG. Expression of MrgprD mRNA is significantly higher than MrgprC11, MrgprA3, Trpv1, and Trpa1. MrgprC11 and MrgprA3 are significantly less abundant than Trpv1 and Trpa1 $\left({ }^{*} p<0.05,{ }^{* *} p<0.01,{ }^{* * *} p<0.001\right.$, ${ }_{* * * *} p 0.0001$, Kruskal-Wallis test with Dunn's multiple comparisons post hoc test, $n=15$ pairs of DRG from N=5 mice). G-I, mRNA expression of MrgprA3, MrgprC11, and MrgprD (and the abundantly expressed Trpv4 as a comparator) in bladder $(\boldsymbol{G})$ detrusor smooth muscle, $(\boldsymbol{H})$ mucosa, and $(\boldsymbol{I})$ primary urothelial cells. Expression of all Mrgprs was similar between the detrusor, urothelial cells, and mucosa (tissue samples from $N=3-5$ mice). Data are mean \pm SEM. 
A

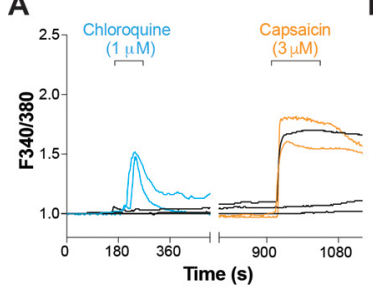

B
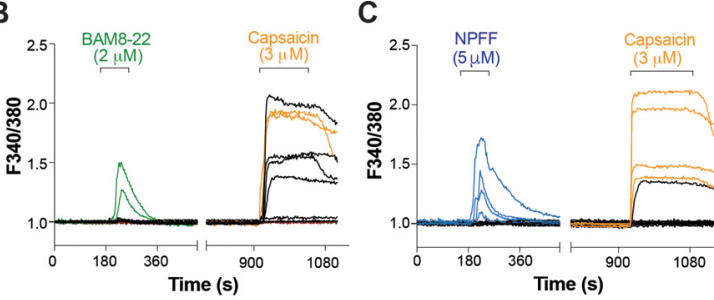

D

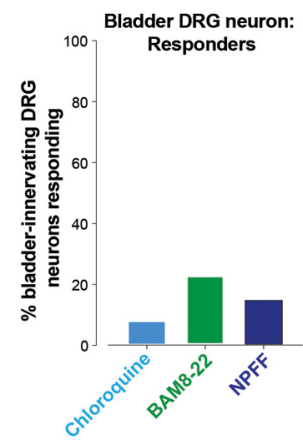

E

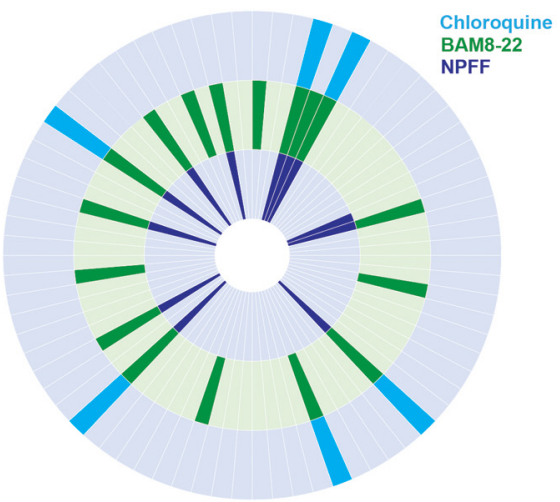

$\mathbf{F}$

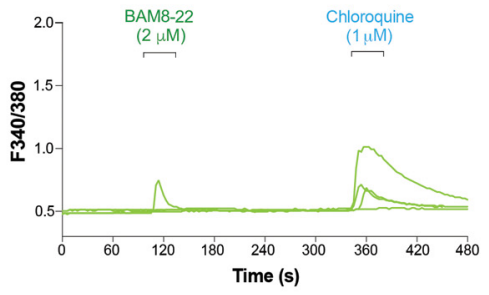

G

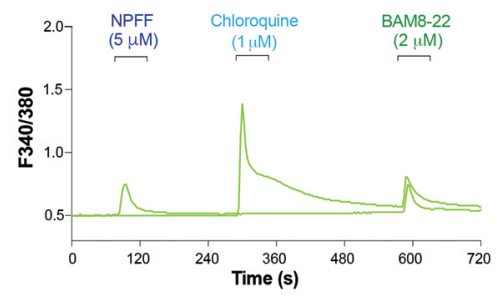

H

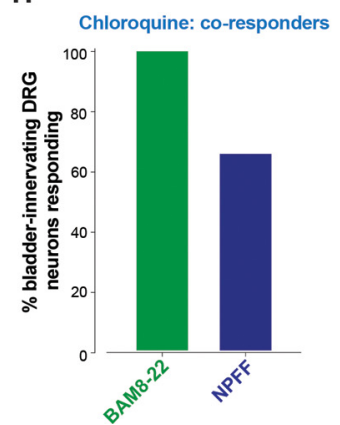

I

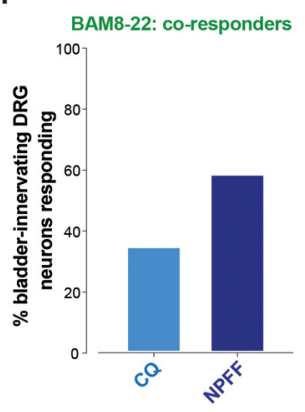

$\mathbf{J}$

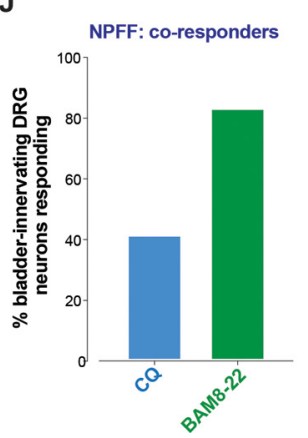

Figure 6. A cohort of bladder-innervating DRG neurons respond to MRGPR agonists. $\boldsymbol{A}$-C, Calcium responses elicited by $(\boldsymbol{A})$ chloroquine $(C Q ; 1 \mu \mathrm{M}),(B)$ BAM8-22 $(2 \mu \mathrm{m})$, and $(\boldsymbol{C})$ NPFF $(5 \mu \mathrm{m})$ in isolated, cultured, retrogradely traced, bladder-innervating LS DRG neurons loaded with fura-2 AM. Capsaicin was added at the end of each experiment to investigate the functional coexpression of TRPV1 with the MRGPRs. Each line indicates an individual neuron. Colored lines indicate bladder DRG neurons responding to MRGPR agonists. Orange capsaicin traces represent bladder neurons that responded to BAM8-22, chloroquine, or NPFF. D, Percentage of bladderinnervating DRG neurons responding to chloroquine (8.1\%: 6 of 74 neurons), BAM8-22 (22.9\%: 17 of 74 neurons), or NPFF (16.2\%: 12 of 74 neurons). $\boldsymbol{E}$, Donut plot showing functional responsiveness of individual neurons to chloroquine, BAM8-22, and NPFF. Bold coloring represents a functional response to an Mrgpr agonist. Chloroquine is represented in the outer ring, with NPFF in the inner ring. Individual neurons are arranged radially, such that functional responsiveness of a single neuron to the Mrgpr agonists can be easily identified running from outside to inside. $\boldsymbol{F}, \boldsymbol{G}$, Example traces showing that bladder-innervating DRG neurons can respond to more than one MRGPR agonist. $\boldsymbol{H}$, All bladder-innervating DRG neurons responding to chloroquine also responded to BAM8-22 (100\%: 6 of 6 neurons), with $66.6 \%$ of chloroquine-responding neurons also responding to NPFF ( 4 of 6 neurons). $I, 35.2 \%$ of BAM8-22 responding neurons also responded to chloroquine (6 of 17 neurons), with $58.8 \%$ responding to NPFF (10 of 17 neurons). J, Of the NPFF responding neurons, 41.6\% (5 of 12 neurons) also responded to chloroquine, with 83.3\% (10 of 12 neurons) also responding to BAM8-22. bladder. Single-cell RT-PCR from retrogradely traced bladder-innervating DRG neurons revealed that, of the 77 individual neurons studied, 14\% expressed MrgprA3, while 38\% expressed MrgprC11 (Fig. 5A,B). In comparison, mRNA transcripts for Trpv1 and Trpa1, key channels involved in bladder afferent function (Daly et al., 2007; DeBerry et al., 2014), were detected in $84 \%$ and $15 \%$ of bladder-innervating DRG neurons, respectively. Interestingly, another pruritogenic Mrgpr, MrgprD, was only expressed in 6\% of bladder-innervating DRG neurons, so was not investigated further with functional studies (Fig. 5A,B). Coexpression analysis revealed that the majority of bladder-innervating neurons that express MrgprA3 coexpress MrgprC11 (90\%) and Trpv1 (81\%), with a smaller proportion coexpressing Trpa1 (36\%) or $\operatorname{MrgprD}$ (18\%) (Fig. 5B,C). Bladder-innervating MrgprC11-expressing DRG neurons commonly coexpressed with Trpv1 (89\%), but less so with MrgprA3 (34\%), TrpA1 (25\%), or MrgprD (7\%) (Fig. 5B,D). Of the few cells that expressed MrgprD, 40\% coexpressed with MrgprA3, MrgprC11, TrpV1, or TrpA1 (Fig. 5B,E). Overall, no bladder-innervating DRG neuron expressed the full repertoire of receptors investigated. Interestingly, the Mrgpr expression profile in bladderinnervating DRG neurons contrasts starkly with that observed in the whole LS DRG, whereby MrgprD mRNA is expressed in the highest abundance (for full details, see Fig. $5 F, p<0.001$, Kruskal-Wallis test, $p=0.0355$ $p<0.0001$, Dunn's multiple comparisons tests). In terms of bladder tissue, MrgprA3, MrgprC11, and $M r g p r D$ were either poorly expressed or were expressed below the detection limit in the detrusor (Fig. 5G, $F_{(1.001,3.002)}=68.43, p=0.0037$, repeated-measures one-way ANOVA, with MrgprA3 vs Trpv4: $p=0.0108$, MrgprC11 vs Trpv4: $p=0.0115$ and MrgprD vs Trpv4: $p=0.0113$, Tukey's multiple comparisons test), the mucosa (Fig. $5 H, F_{(1.000,3.000)}=53.86$, $p=0.0052$ repeated-measures one-way ANOVA, with MrgprA3 vs Trpv4: $p=0.0158$, MrgprC11 vs Trpv4: $p=0.0158$ and MrgprD vs Trpv4: $p=0.0158$, Tukey's multiple comparisons test), or primary urothelial cells (Fig. $5 I, F_{(3,14)}=1109$, $p<0.001$, one-way ANOVA, with MrgprA3 vs Trpv4: $p<0.0001$, MrgprC11 vs Trpv4: $p<0.0001$ and MrgprD vs Trpv4: $p<0.0001$, Tukey's multiple comparisons test), particularly when compared with an abundantly expressed target, such as Trpv4 (Everaerts et al., 2010a,b). Mrgpr mRNA expression was $\sim 1000$ $100,000 \times$ lower than expression of $\operatorname{Tr} p v 4$. Overall, these findings indicate that the pruritogenic receptors MrgprC11 and MrgprA3 are expressed on sensory neurons innervating the bladder, and their expressions profile correlates well with 
our observations that subpopulations of bladder afferents display mechanical hypersensitivity following application of the respective Mrgpr agonists. The relatively low expression of Mrgprs in bladder tissue correlates well with our findings that Mrgpr agonists do not affect bladder compliance.

\section{Mrgpr agonists activate subpopulations of bladder- innervating DRG neurons}

In order to confirm the results of our single-cell RT-PCR at a functional level, and to investigate the roles of Mrgpr agonists in activating bladder-innervating DRG neurons, we measured intracellular calcium $\left(\left[\mathrm{Ca}^{2+} \mathrm{i}\right)\right.$ using fura-2 AM in response to application of chloroquine, BAM8-22, and NPFF. Overall, calcium imaging of dissociated bladderinnervating DRG neurons revealed that individual application of chloroquine (Fig. 6A), BAM8-22 (Fig. 6B), or NPFF (Fig. $6 C$ ) resulted in activation of subpopulations of bladder-innervating DRG neurons. Overall, 8.1\%, 22.9\%, and $16.2 \%$ of bladder-innervating LS DRG neurons were activated by chloroquine, BAM8-22, and NPFF, respectively (Fig. $6 D, E)$. In series application of multiple Mrgpr agonists demonstrated functional coexpression of MrgprA3 and MrgprC11 in populations of bladderinnervating DRG neurons (Fig. $6 E-J$ ), supporting our single-cell RT-PCR data suggesting colocalization of MrgprC11 and MrgprA3 in bladder-innervating DRG neurons.

To further investigate the role of Mrgprs in modulating bladder sensation, we used whole-cell patch-clamp electrophysiology of retrogradely traced bladder-innervating DRG neurons. In these studies, we coapplied the Mrgpr agonists chloroquine, BAM8-22, and NPFF at the same time as an itch cocktail, as used in some of our bladder afferent recording studies (Fig. 7A). We found that this mixture caused significant hyperexcitability in a subpopulation (44\%) of bladder-innervating DRG neurons, as indicated by a significant decrease in the rheobase, or amount of current required to fire an action potential (Fig. 7Ai: responders: $t_{(6)}=4.583, p=0.0038$, paired $t$ test, Fig. 7Aii,Aiii). These changes in responder neurons were also accompanied by a significant increase in the number of action potentials fired at $2 \times$ rheobase (Fig. $7 \mathrm{Bi}$ : responders: $t_{(6)}=4.044, p=0.0068$, paired $t$ test, Fig. 7 Bii, Biii). In comparison, the neuronal excitability of another population of bladder-innervating DRG neurons was unaffected by the Mrgpr agonist mixture (Fig. $7 A i$ : nonresponders: $t_{(8)}=1.155$,

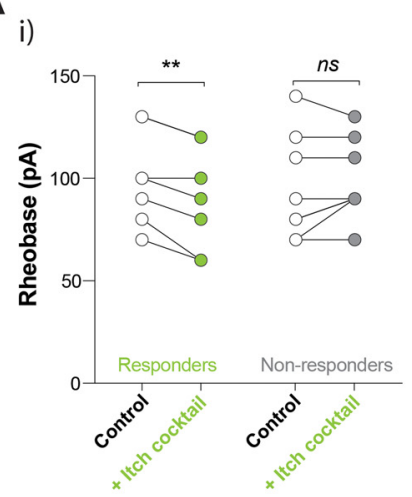

ii)
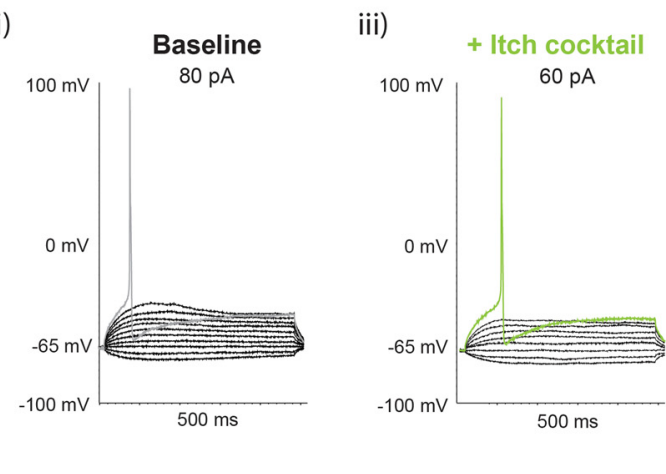

B

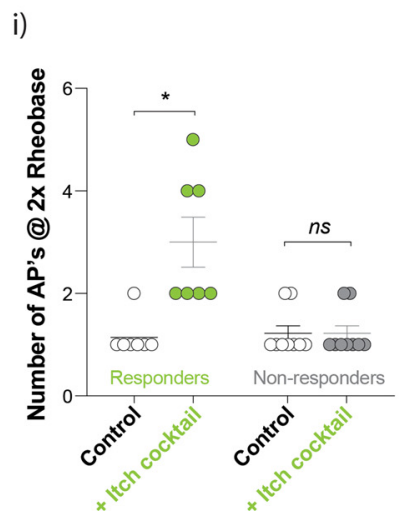

ii)

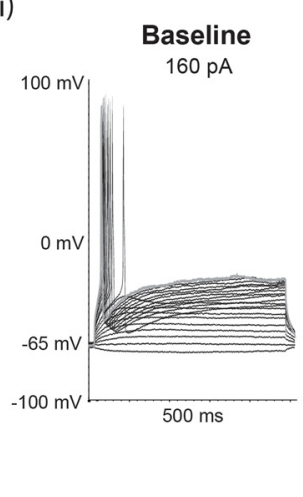

iii)

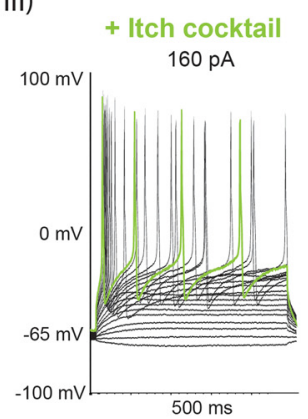

Figure 7. MRGPR agonists induce neuronal hyperexcitability in a cohort of bladder-innervating DRG neurons. $\boldsymbol{A}$, Whole-cell patch-clamp recordings of retrogradely traced bladder-innervating LS DRG neurons. $\boldsymbol{A i}$, The Mrgpr agonist mixture, referred to in the figure as an itch cocktail significantly decreases the amount of current required to elicit an action potential in a subset (7 of 16) of bladder-innervating DRG neurons (rheobase; ${ }^{* *} p<0.01$, paired $t$ test, $n=16$ neurons from $N=5$ mice). Another population of bladder-innervating DRG neurons ( 9 of 16) were unaffected by the itch cocktail (not significant, $p>0.05$; paired Student $t$ test, $n=9$ neurons from $N=5$ mice). Aii, Original recording of membrane voltage from a single bladder-innervating was required to elicit an action potential under control conditions (gray trace). Aiii, Perfusion with the itch cocktail consisting of chloroquine $(1 \mu \mathrm{m})$, NPFF $(5 \mu \mathrm{m})$, and BAM8-22 $(2 \mu \mathrm{m})$ for 2 min onto the same DRG neuron reduced the amount current to elicit an action potential (green trace), indicating increased neuronal excitability in this "responding" neuron. $\boldsymbol{B} \boldsymbol{i}$, Application of the itch cocktail significantly increases the number of action potentials fired at a current twice $(2 \times)$ the rheobase in a subset $(7$ of $16 ; 44 \%)$ of bladder-innervating DRG neurons $\left({ }^{*} p<0.05\right.$, Wilcoxon matched-pairs signed rank test, $n=7$ s from $N=5$ mice). Other bladder-innervating DRG neurons were unaffected by the itch cocktail ( 9 of 16; $56 \%$, not significant, $p>0.05$, paired $t$ test, $n=16$ neurons from $N=5$ mice). Bii, Original whole-cell current-clamp recordings from a single bladder-innervating DRG neuron during current-clamp protocols. Each line indicates a $10 \mathrm{pA}$ increase in current. In this example, an $80 \mathrm{pA}$ current was required to elicit an action potential under control conditions, with $160 \mathrm{pA}$ representing $2 \times$ rheobase (gray trace). Biii, Following a 2 min perfusion with the itch cocktail, the same DRG neuron dramatically increased the number of action potentials at $2 \times$ rheobase (green trace), indicating an increase in neuronal excitability. $\boldsymbol{B i}$, Data are mean \pm SEM. LS, Lumbosacral; AP's, action potentials.

$p=0.281$, paired $t$ test, Fig. $7 B i$ : nonresponders: each row has the same difference).

Peripheral activation of mrgprs in the bladder results in activation of dorsal horn neurons within the lumbosacral spinal cord

Based on our findings that activation of MrgprA3 and MrgprC11 sensitized the response of a proportion of bladder afferents ex vivo, we hypothesized that in vivo intrabladder infusion of Mrgpr agonists should correspondingly enhance bladder signaling into the dorsal horn of the spinal cord. Infusion of saline into the bladder resulted in pERK immunoreactivity (pERKIR) in neurons within the LS spinal cord dorsal horn because of low-level bladder distension (Fig. $8 A, B$ ). In LS spinal segments, pERK-IR was seen in the SDH (Fig. $8 A i i, B$ ), the SPN (Fig. 
A

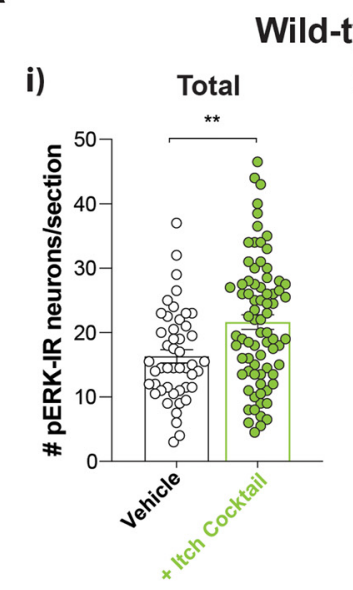

\section{Wild-type}

ii)

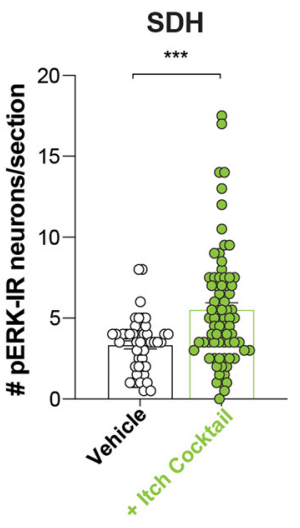

iii)

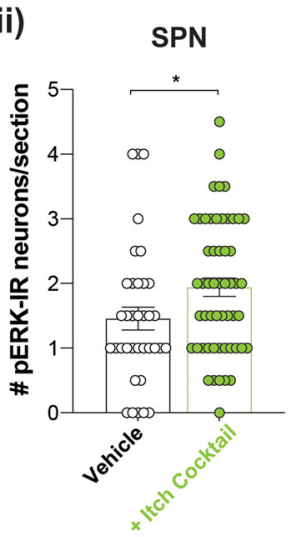

iv) DGC

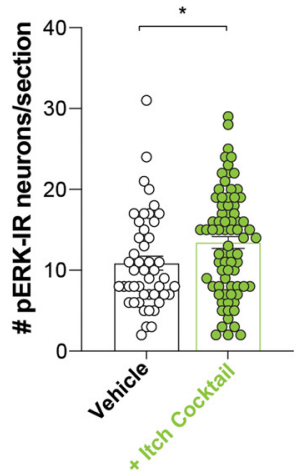

B

B

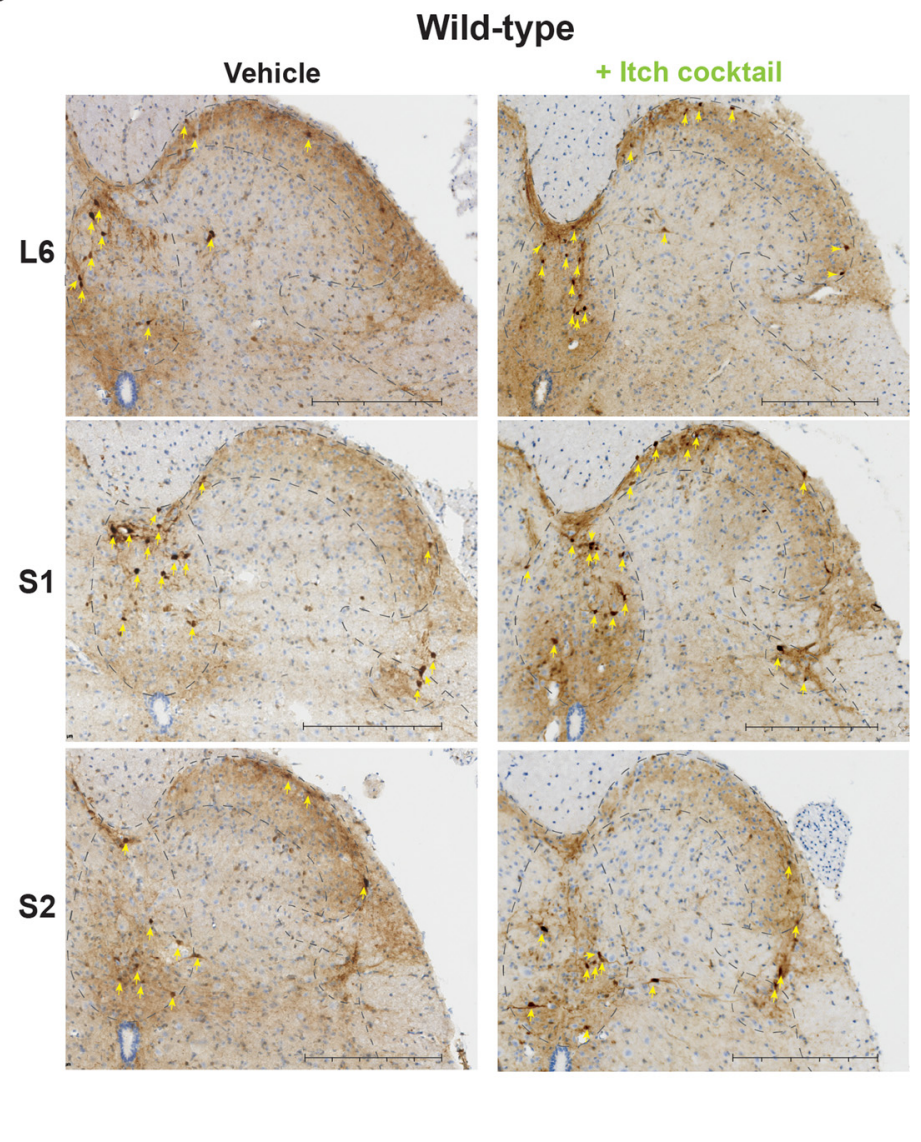

C

Mrgpr cluster $\%$

i)

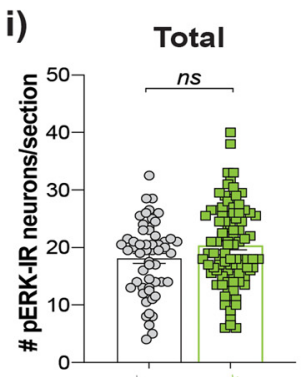

iii)

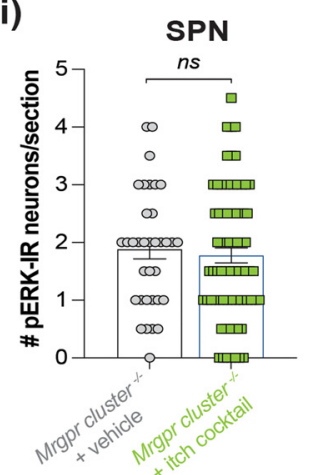

ii)

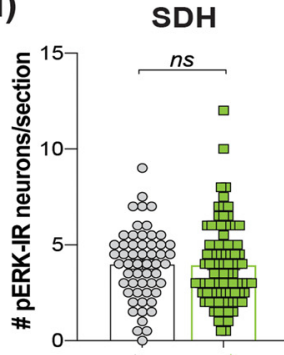

iv)
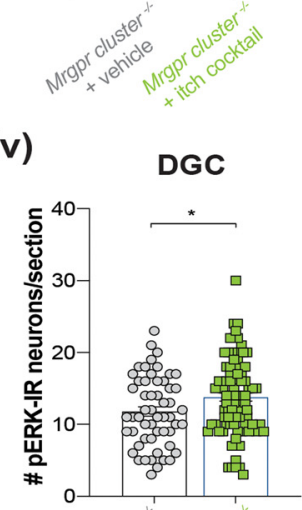

S2

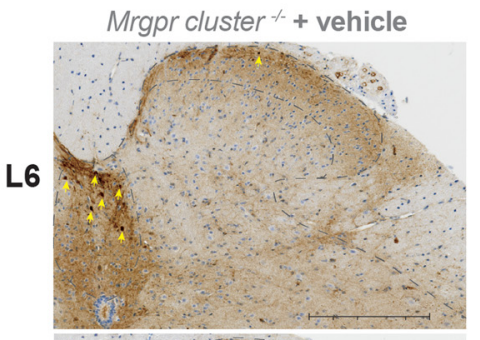

S1

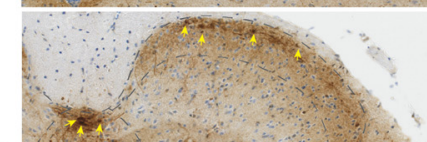

S1
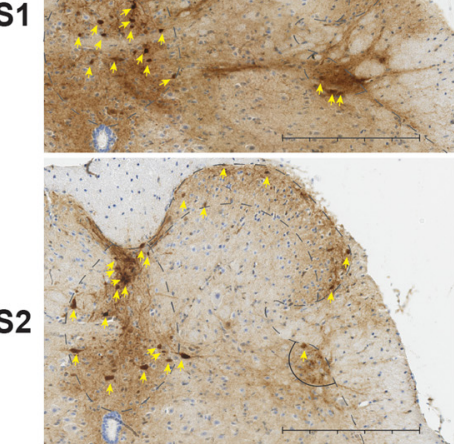

Mrgpr cluster ${ }^{-/}$
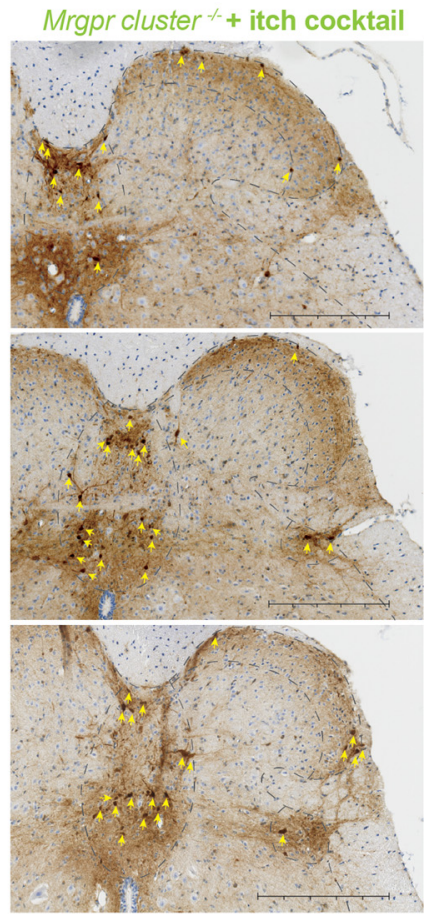

Figure 8. In vivo intrabladder administration of an MRGPR agonist itch cocktail results in enhanced activation of dorsal horn neurons within the lumbosacral spinal cord. $\boldsymbol{A}$, pERK-IR was measured to determine signaling within the lumbosacral (LS; L6-S2) spinal cord in response to intrabladder administration of saline or an itch cocktail of MRGPR agonists (chloroquine: $10 \mu \mathrm{M}$, BAM8-22: $20 \mu \mathrm{m}$, NPFF: $5 \mu \mathrm{m}$ ). Ai, pERK-IR was significantly increased in the LS dorsal horn of WT mice administered the itch cocktail compared with those administered saline $\left({ }^{* *} p<0.01\right.$, 
$8 A i i i, B$ ), and the DGC (Fig. $8 A i v, B$ ). Infusion of the itch cocktail by coapplying chloroquine, BAM8-22, and NPFF into the bladder resulted in significantly greater total numbers of pERK-IR neurons in the LS spinal cord relative to saline infusion (Fig. $8 \mathrm{~A} i$; $p=0.0025$, saline: $16.34 \pm 1.030$ vs itch cocktail: $21.65 \pm 1.125$, Mann-Whitney test, Fig. $8 B$ ). Furthermore, significant increases in pERK-IR neurons were observed across the SDH (Fig. 8Aii: $p=0.0003$, saline: $3.340 \pm 0.249$ vs itch cocktail: $5.526 \pm 0.411$, Mann-Whitney test, Fig. $8 B$ ), SPN (Fig. 8 Aiii: $p=0.0255$, saline: $1.458 \pm 0.178$ vs itch cocktail: $1.944 \pm 0.140$, Mann-Whitney test, Fig. $8 B$ ), and the DGC (Fig. 8Aiv: $p=0.0219$, saline: $10.89 \pm 0.860$ vs itch cocktail: $13.45 \pm 0.743$, Mann-Whitney test, Fig. 8B), indicating that Mrgpr-evoked bladder afferent hypersensitivity is relayed into the spinal cord and enhances sensory signaling. To confirm that the effects we observed with Mrgpr agonists are mediated via Mrgpr activation, we performed the same in vivo experiments with Mrgpr-cluster $\Delta^{-1-}$ mice. Comparing Mrgpr agonist and vehicle-treated Mrgprcluster $\Delta^{-1-}$ mice, we found no differences in the total number of pERK-IR neurons in the LS spinal cord (Fig. 8Ci: $p=0.105$, saline: $18.14 \pm 0.854$ vs itch cocktail: $20.36 \pm 0.739$, MannWhitney test, Fig. $8 D$ ), the SDH (Fig. 8Cii: $p=0.5172$, saline: $3.982 \pm 0.251$ vs itch cocktail: $3.929 \pm 0.217$, Mann-Whitney test, Fig. $8 D$ ), and the SPN (Fig. 8Ciii: $p=0.584$, saline: $1.886 \pm 0.171$ vs itch cocktail: $1.777 \pm 0.131$, Mann-Whitney test, Fig. $8 D$ ), with a small but significant increase in the DGC (Fig. 8Civ: $p=0.031$, saline: $11.80 \pm 0.654$ vs itch cocktail: $13.80 \pm 0.546$, Mann-Whitney test, Fig. $8 D$ ).

\section{Baseline bladder afferent responses and signaling within the spinal cord to bladder distension are unaltered in Mrgpr-

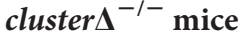

In order to determine whether Mrgprs contribute to bladder afferent responses to distension at baseline, we compared responses in WT and Mrgpr-cluster $\Delta^{-1-}$ littermate mice. Ex vivo bladder afferent firing to bladder distension with saline showed that responses were indistinguishable between WT and Mrgprcluster $^{-1-}$ mice (Fig. 9A, $F_{(15,272)}=0.037, p>0.999$, two-way repeated-measures ANOVA). Similarly, following saline bladder distension in vivo, the numbers of pERK-IR neurons within the LS spinal cord were not significantly different between WT and Mrgpr-cluster $\Delta^{-1-}$ mice (Fig. $9 B, t_{(100)}=1.353, p=0.179$, unpaired $t$ test; Fig. $9 C, t_{(100)}=1.801, p=0.074$, unpaired $t$ test;

\footnotetext{
Mann-Whitney test, $n=47-76$ sections from spinal regions $\mathrm{L} 6-\mathrm{S} 2 ; N=5$ mice). Aii-Aiv, WT mice administered the intrabladder itch cocktail displayed significantly increased pERK-IR in specific regions of the dorsal horn, including the (Aii) SDH, (Aiii) SPN, and (Aiv) DGC $\left({ }^{*} p<\right.$ $0.05,{ }^{* * *} p<0.001$, Mann-Whitney tests). Data are presented as numbers of pERK-IR neurons/section of the LS spinal cord, with a minimum of 6 sections/mouse. $\boldsymbol{B}$, Representative pERK-labeled LS spinal cord images at each spinal level (L6-S2) from mice that had bladders infused with either saline or an MRGPR agonist itch cocktail. Yellow arrows indicate pERK-IR neurons. Scale bar, $250 \mu \mathrm{m}$. Ci, In contrast to WT mice, pERK-IR was not significantly different across the whole $L S$ dorsal horn of Mrgpr-cluster $\Delta^{-/-}$mice administered either intrabladder saline or the MRGPR agonist itch cocktail (not significant, $p>0.05$, Mann-Whitney test, $n=55-91$ sections from $N=5$ mice). Cii, (iiii, pERK-IR was not significantly altered in the (Cii) SDH, (Ciii) nor the SPN (not significant, $p>0.05$, Mann-Whitney tests, $N=5$ mice). Civ, Following the itch cocktail infusion, there was a small but significant increase in pERK-IR in the DGC that remained in Mrgpr-cluster $\Delta^{-1-}$ mice $\left({ }^{*} p<0.05\right.$; Mann-Whitney tests, $N=5$ mice). Data are presented as numbers of $p E R K-I R$ neurons/section of the $L S$ spinal cord, with a minimum of 6 sections/mouse. D, Representative pERK-labeled LS spinal cord images at each spinal level (L6-S1) from Mrgpr-cluster $\Delta^{-1-}$ mice that had bladders infused with either saline or the MRGPR agonist itch cocktail. Yellow arrows indicate pERK-IR neurons. Scale bars, $250 \mu \mathrm{m}$. Data are mean \pm SEM.
}

Fig. $9 D, t_{(69)}=1.724, p=0.089$, unpaired $t$ test; Fig. $9 E, t_{(100)}=$ $0.850, p=0.396$, unpaired $t$ test). Overall, these findings suggest that Mrgprs are not responsible for bladder afferent mechanosensitivity per se, but rather have a prominent role in sensitizing bladder afferent responsiveness to distension following activation by pruritogens.

\section{Discussion}

The ability of the somatosensory nervous system to easily differentiate stimuli, such as pruritogens or algogens, is essential as it evokes a tailored response, such as scratching or withdrawal of a limb. As a consequence, there is significant functional and anatomic diversity of somatosensory afferent nerves. In contrast, sensory discrimination in the bladder is more limited in scope, ranging from fullness to urge and finally pain (de Groat et al., 2015). Therefore, the differentiation of distinct sensory stimuli (inflammation, infection, irritation) is less important if the overarching physiological response remains the same: increased voiding to rapidly remove irritants from the bladder. As such, mechanosensitive afferents innervating the bladder are less diverse than cutaneous afferents. The majority of bladder afferent neurons are embedded within the detrusor smooth muscle (de Groat et al., 2015; Spencer et al., 2018; Grundy et al., 2019a, b), and are broadly tuned to allow the detection of a variety of mechanical and chemical stimuli (Rong et al., 2002; Zagorodnyuk et al., 2006, 2007; de Groat and Yoshimura, 2009a; Grundy et al., 2019b). Therefore, the excitability of these afferents is key in providing appropriate sensory information in response to stimuli, including bladder filling and emptying. Understanding the mechanisms responsible for regulating bladder afferent mechanosensitivity is therefore crucial to the development of novel treatments for the chronic sensory symptoms associated with bladder hypersensitivity and dysfunction, including urinary urgency, frequency, and pelvic pain. In this study, we discovered a novel role in bladder sensory afferent pathways for irritant sensing mechanisms mediated via MrgprC11 and MrgprA3.

Consistent with previous reports that Mrgpr agonists are able to excite isolated peripheral sensory neurons (Q. Liu et al., 2009; Castro et al., 2019; Tseng et al., 2019), we showed, using patchclamp recordings, that chloroquine, BAM8-22, and NPFF activated and induced neuronal hyperexcitability in subpopulations (44\%) of bladder-innervating DRG neurons. In calcium imaging experiments, we observed some functional overlap between responses to chloroquine and BAM8-22, which has been previously described in afferents innervating the skin (Q. Liu et al., 2009) and colon (Castro et al., 2019). Using single-cell RT-PCR of bladder DRG neurons, we revealed expression and coexpression of Mrgprs in bladder-innervating neurons. The majority of neurons expressing MrgprC11 also contained MrgprA3, consistent with previous reports of colocalization in other sensory pathways (Dong et al., 2001; Zylka et al., 2003; Han et al., 2013; Castro et al., 2019).

High colocalization of MrgprA3 and MrgprC11 was also observed with Trpv1 in bladder DRG neurons, either on an mRNA level with single-cell RT-PCR, or functionally with bladder neurons exhibiting calcium transients in response to chloroquine, BAM8-22, NPFF, and the TRPV1 agonist capsaicin. This is expected as the majority of bladder-innervating DRG neurons express Trpv1 (La et al., 2011; Dang et al., 2013; Forrest et al., 2013), while TRPV1 is a key integrator of histamine and $\mathrm{P}_{2} \mathrm{X}_{3}$ dependent bladder afferent activation (Grundy et al., 2018a, 


\section{A}

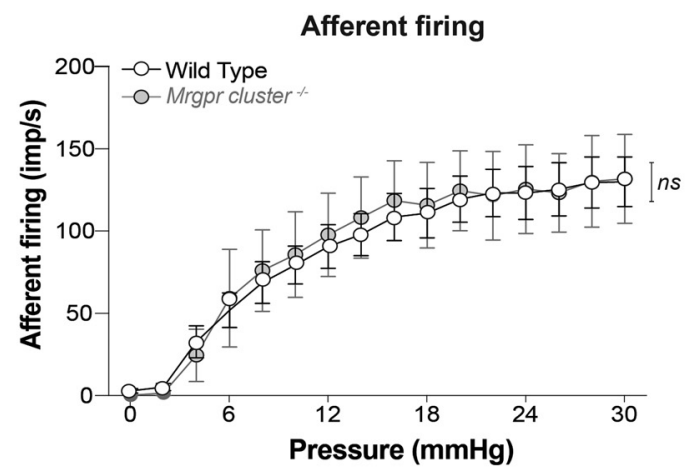

B

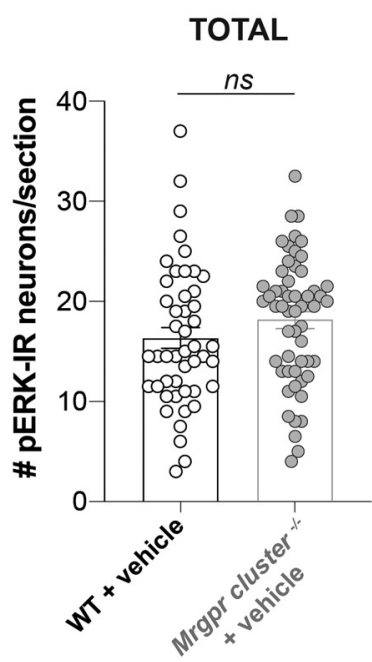

C

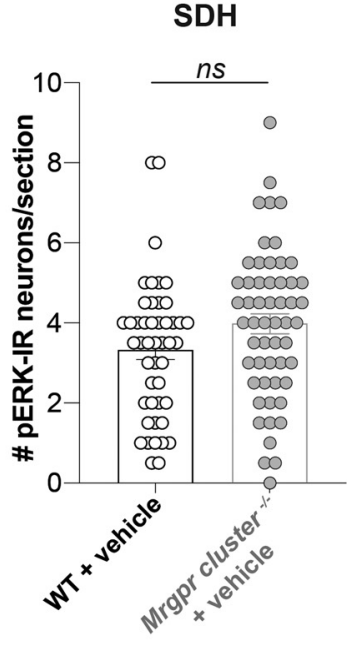

D

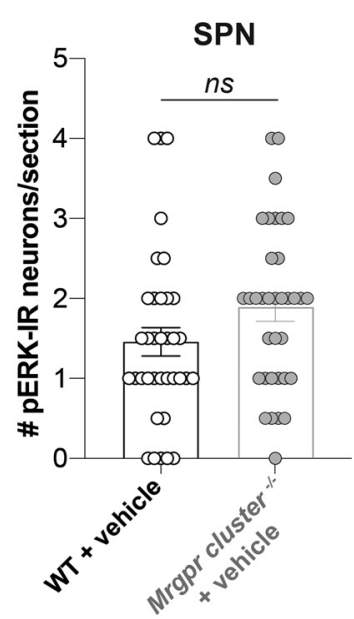

E

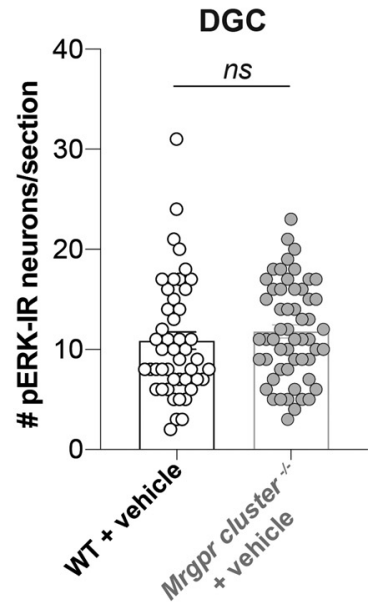

Figure 9. Mrgpr-cluster $\Delta^{-1-}$ mice do not display altered baseline responses to bladder distension. $\boldsymbol{A}$, Group data from ex vivo recordings from WT mice and age-matched Mrgprcluster $\Delta^{-/-}$mice. There was no significant difference in the mechanosensitivity of bladder afferents in response to graded bladder distension (0-30 $\mathrm{mmHg}$ ) between WT and Mrgpr-

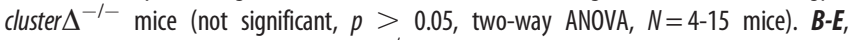
Compared with WT mice, Mrgpr-cluster $\Delta^{-1-}$ mice do not display altered neuronal activation within the dorsal horn of LS spinal cord following intrabladder vehicle administration. pERKIR was measured to determine signaling in the lumbosacral (LS; L6-S2) spinal cord in response to saline instillation in WT and age-matched Mrgpr-cluster $\Delta^{-1-}$ mice. There was no difference in $(\boldsymbol{B})$ the total number of pERK-IR neurons identified within the LS dorsal horn in response to saline infusion into the bladder between WT and Mrgpr-cluster $\Delta^{-1-}$ mice. In terms of specific regions of the $L S$ dorsal horn, there were significant differences between
2020). These findings are consistent with the literature suggesting that MrgprA3-positive neurons are in a subpopulation of Trpv1-positive neurons (Han et al., 2013; Usoskin et al., 2015; Meixiong and Dong, 2017). However, these mechanisms in the bladder, where only $15 \%$ of bladder neurons express Trpa1, appear to contrast with those in the colon and skin. For example, Mrgpr agonist-induced mechanical hypersensitivity to colorectal distension is not evoked in Trpa1 ${ }^{-1-}$ mice (Castro et al., 2019). In the skin, TRPA1 has been identified as the downstream target of both MrgprA3 and MrgprC11, whereby Trpa1 ${ }^{-1-}$ mice display little to no scratching in response to chloroquine or BAM8-22, with functional coupling of MrgprA3 or MrgprC11 to TRPA1 occurring via G $\beta \gamma$ and PLC signaling, respectively (Wilson et al., 2011).

Differences are also apparent between bladder and cutaneous neurons when interrogating the role of MrgprD. Within cutaneous populations, two distinct pruritogenic subpopulations of DRG neurons have been identified: those expressing MrgprA3, $\operatorname{MrgprC11}$, and histamine receptor $1\left(H_{1} R\right)$, and those expressing MrgprD (Q. Liu et al., 2009, 2012; Usoskin et al., 2015). MrgprD, initially postulated to play an important nociceptive role, is now known to mediate itch induced by the amino acid $\beta$-alanine (Shinohara et al., 2004; Q. Liu et al., 2012). Despite high MrgprD expression in whole LS DRG, only 6\% of bladder-innervating DRG neurons expressed MrgprD, which showed equal coexpression (40\%) with MrgprC11 and MrgprA3. This suggests a far more prominent role for MrgprC11 and MrgprA3 than MrgprD in bladder sensory function.

In keeping with this reasoning, we show that chloroquine, BAM8-22, and NPFF enhanced the mechanosensitivity of subpopulations (20\%-41\%) of WT pelvic bladder afferents to bladder distension, which did not occur in experiments with Mrgprcluster $\Delta^{-1-}$ mice. The proportion of WT afferents responding to these Mrgpr agonists correlated reasonably well with the proportion of isolated lumbosacral bladder neurons responding to MrgprA3 (8\%) and MrgprC11 (23\%) agonists in calcium imaging experiments and the percentage of lumbosacral bladder DRG neurons expressing mrgprA3 (14\%) and $m r g p r C 11$ (38\%). Differences between the exact proportions may be because of culture times, $4 \mathrm{~h}$ was for single-cell RT-PCR and 18-48 $\mathrm{h}$ was for calcium imaging. A recent study identified that only $4 \%$ of bladder neurons express mrgpra3; however, they were isolated from both thoracolumbar and lumbosacral DRG, and no functional responses were investigated (Meerschaert et al., 2020). Differences between these studies are likely because of the location of the neurons and the specific culture conditions, including using different enzymes and media supplementation.

Mrgpr mRNA expression in bladder tissue was extremely low or below the detection limit, explaining why intrabladder instillation of Mrgpr agonists did not affect bladder compliance. These findings are in keeping with a number of studies showing that these Mrgprs are expressed almost exclusively in sensory structures, including cell bodies of sensory neurons located in the DRG, trigeminal, and jugular ganglia (Dong et al., 2001; Han et al., 2013, 2018; Meixiong and Dong, 2017; Castro et al., 2019).

We found that Mrgpr agonists evoked bladder afferent mechanical hypersensitivity across a broad range of distension

$\leftarrow$

WT and Mrgpr-Cluster $\Delta^{-1-}$ mice in the number of pERK-IR neurons following intrabladder administration within the $(C)$ SDH, (D) SPN, and (E) DGC regions of the dorsal horn (not significant, $p>0.05$, unpaired $t$ tests, $N=5$ mice/group). Data are mean \pm SEM. 
pressures. Unlike other irritant and inflammatory stimuli, such as histamine or inflammatory cytokines, Mrgpr agonist incubation did not recruit silent afferents to become mechanosensitive (Brierley et al., 2020; Grundy et al., 2020). This is an important distinction as silent afferents represent a discrete functional subclass of bladder afferents, that after becoming sensitized acquire an ability to respond to "high-threshold" or "nociceptive" mechanosensory stimuli (Prato et al., 2017; Zhang et al., 2019; Brierley et al., 2020). These findings are consistent with MrgprA3- and MrgprC11-positive neurons that have previously been reported as being responsive to mechanical stimuli, including probing of cutaneous receptive fields (Han et al., 2013). Furthermore, a recent study investigating the role of Mrgprs in colonic afferent mechanosensitivity found that MrgprA3- and MrgprC11-positive fibers are also sensitive to mechanical stimulation (Castro et al., 2019). Mrgpr-positive cutaneous nerve endings have been reported to exclusively innervate the epidermis (Han et al., 2013); however, this does not appear to be the case in the bladder. While some mechanosensitive afferents innervate the bladder mucosa, an equivalent to the epidermis, the majority of mechanosensitive bladder afferents are found innervating the bladder detrusor (Zagorodnyuk et al., 2007; Spencer et al., 2018), and we observed significant hypersensitivity of these distension sensitive afferents to Mrgpr agonists.

The homogeneity of bladder sensory pathways is further reflected in their activation of spinal cord circuits in response to bladder distension, which, unlike the distinct organization of primary afferent circuits that occurs in the spinal cord for processing specific somatosensory stimuli (Todd, 2010), appears graded only to the level of peripheral stimuli (Grundy et al., 2018a). As such, an increase in the intensity of the peripheral afferent signal corresponds to increased activation of second-order neurons that project within multiple visceral sensory pathways to regulate networks responsible for both micturition and sensation, rather than any obvious sensation-specific activation. Accordingly, we found that Mrgpr activation of peripheral afferents in the bladder in vivo resulted in significant increases in the number of pERKIR neurons in all regions of the lumbosacral dorsal horn that receive input from mechanosensitive afferents (Grundy et al., $2019 b$ ). This included increased numbers of neurons within the SDH, DGC, and SPN. As such, we would expect that the physiological consequence of an Mrgpr-induced increase in afferent firing would be similar to other stimuli that evoke an increase in primary afferent excitability from the bladder: urinary urgency and urinary frequency.

Interestingly, we observed no difference in bladder afferent mechanosensitivity nor activation of central pathways in the spinal cord to distension with saline between Mrgpr-cluster $\Delta^{-1-}$ mice and WT mice. Similar observations have been made before in other sensory pathways, where Mrgpr-cluster $\Delta^{-1-}$ mice do not show deficits in cutaneous mechanosensation (Q. Liu et al., 2009). Furthermore, in the colon, Mrgpr-cluster $\Delta^{-1-}$ mice had similar visceromotor responses to WT mice (Castro et al., 2019). Overall, these data suggest that endogenous activators of MrgprC11 and MrgprA3 are not tonically released from the bladder to set the baseline tone of bladder afferent mechanosensitivity; however, it has yet to be established whether compensatory changes occur in Mrgpr-cluster $\Delta^{-1-}$ mice to maintain normal function.

While no known endogenous MrgprA3 agonists have been documented, endogenous MrgprC11 agonists include BAM8-22, proenkephalin A, SLIGRL, NPFF, and cathepsin S, which may be found in a variety of different cell types, including fibroblasts, keratinocytes, mast cells, macrophages, and microglia (M. G. Lee et al., 2008; Q. Liu et al., 2009; Slominski et al., 2011; T. Liu and Ji, 2013; LaMotte et al., 2014; Reddy et al., 2015; MontagueCardoso and Malcangio, 2020). Whether or not Mrgprs or their endogenous agonists are upregulated in conditions of bladder pathophysiology is the subject of future research.

In conclusion, we have shown that MrgprC11 and MrgprA3 are present and functional on mechanosensitive bladder afferents, and their activation induces mechanical hypersensitivity and increased activation of sensory circuits within the spinal cord. Enhanced sensation via an increase in mechanosensitivity may be a fundamental protective mechanism and relate more specifically to bladder disorders associated with urgency and frequency in the absence of pain, such as overactive bladder.

\section{References}

Abrams P, Cardozo L, Fall M, Griffiths D, Rosier P, Ulmsten U, van Kerrebroeck P, Victor A, Wein A, Standardisation Sub-committee of the International Continence Society (2002) The standardisation of terminology of lower urinary tract function: report from the Standardisation Subcommittee of the International Continence Society. Neurourol Urodyn 21:167-178.

Andersson KE (2019) TRP channels as lower urinary tract sensory targets. Med Sci (Basel) 7:67.

Bellono NW, Bayrer JR, Leitch DB, Castro J, Zhang C, O'Donnell TA, Brierley SM, Ingraham HA, Julius D (2017) Enterochromaffin cells are gut chemosensors that couple to sensory neural pathways. Cell 170:185198.e116.

Brierley SM, Jones RC 3rd, Gebhart GF, Blackshaw LA (2004) Splanchnic and pelvic mechanosensory afferents signal different qualities of colonic stimuli in mice. Gastroenterology 127:166-178.

Brierley SM, Goh KG, Sullivan MJ, Moore KH, Ulett GC, Grundy L (2020) Innate immune response to bacterial urinary tract infection sensitises high-threshold bladder afferents and recruits silent nociceptors. Pain 161:202-210.

Castro J, Harrington AM, Garcia-Caraballo S, Maddern J, Grundy L, Zhang J, Page G, Miller PE, Craik DJ, Adams DJ, Brierley SM (2017) $\alpha$-Conotoxin Vc1.1 inhibits human dorsal root ganglion neuroexcitability and mouse colonic nociception via GABAB receptors. Gut 66:10831094.

Castro J, Harrington AM, Lieu T, Garcia-Caraballo S, Maddern J, Schober G, O’Donnell T, Grundy L, Lumsden AL, Miller P, Ghetti A, Steinhoff MS, Poole DP, Dong X, Chang L, Bunnett NW, Brierley SM (2019) Activation of pruritogenic TGR5, MrgprA3, and MrgprC11 on colon-innervating afferents induces visceral hypersensitivity. JCI Insight 4:e131712.

Christianson JA, Liang R, Ustinova EE, Davis BM, Fraser MO, Pezzone MA (2007) Convergence of bladder and colon sensory innervation occurs at the primary afferent level. Pain 128:235-243.

Daly D, Rong W, Chess-Williams R, Chapple C, Grundy D (2007) Bladder afferent sensitivity in WT and TRPV1 knockout mice. J Physiol 583:663674.

Dang K, Bielefeldt K, Gebhart GF (2013) Cyclophosphamide-induced cystitis reduces ASIC channel but enhances TRPV1 receptor function in rat bladder sensory neurons. J Neurophysiol 110:408-417.

de Groat WC, Yoshimura N (2009a) Afferent nerve regulation of bladder function in health and disease. In: Sensory nerves (Canning BJ, Spina D, eds), pp 91-138. Berlin: Springer.

de Groat WC, Yoshimura N (2009b) Afferent nerve regulation of bladder function in health and disease. Handb Exp Pharmacol 194:91-138.

de Groat WC, Griffiths D, Yoshimura N (2015) Neural control of the lower urinary tract. Compr Physiol 5:327-396.

DeBerry JJ, Schwartz ES, Davis BM (2014) TRPA1 mediates bladder hyperalgesia in a mouse model of cystitis. Pain 155:1280-1287.

Dong X, Han S, Zylka MJ, Simon MI, Anderson DJ (2001) A diverse family of GPCRs expressed in specific subsets of nociceptive sensory neurons. Cell 106:619-632. 
Everaerts W, Vriens J, Owsianik G, Appendino G, Voets T, De Ridder D, Nilius B (2010a) Functional characterization of transient receptor potential channels in mouse urothelial cells. Am J Physiol Renal Physiol 298: F692-F701.

Everaerts W, Zhen X, Ghosh D, Vriens J, Gevaert T, Gilbert JP, Hayward NJ, McNamara CR, Xue F, Moran MM, Strassmaier T, Uykal E, Owsianik G, Vennekens R, De Ridder D, Nilius B, Fanger CM, Voets T (2010b) Inhibition of the cation channel TRPV4 improves bladder function in mice and rats with cyclophosphamide-induced cystitis. Proc Natl Acad Sci USA 107:19084-19089.

Forrest S, Osborne P, Keast J (2013) Characterization of bladder sensory neurons in the context of myelination, receptors for pain modulators, and acute responses to bladder inflammation. Front Neurosci 7:206.

Gao YJ, Ji RR (2009) c-Fos and pERK, which is a better marker for neuronal activation and central sensitization after noxious stimulation and tissue injury? Open Pain J 2:11-17.

Grundy L, Caldwell A, Brierley SM (2018a) Mechanisms underlying overactive bladder and interstitial cystitis/painful bladder syndrome. Front Neurosci 12:931.

Grundy L, Erickson A, Caldwell A, Garcia-Caraballo S, Rychkov G, Harrington A, Brierley SM (2018b) Tetrodotoxin-sensitive voltage-gated sodium channels regulate bladder afferent responses to distension. Pain 159:2573-2584.

Grundy L, Chess-Williams R, Brierley SM, Mills K, Moore KH, Mansfield K, Rose'Meyer R, Sellers D, Grundy D (2018c) NKA enhances bladder-afferent mechanosensitivity via urothelial and detrusor activation. Am J Physiol Renal Physiol 315:F1174-F1185.

Grundy L, Harrington AM, Castro J, Garcia-Caraballo S, Deiteren A, Maddern J, Rychkov GY, Ge P, Peters S, Feil R, Miller P, Ghetti A, Hannig G, Kurtz CB, Silos-Santiago I, Brierley SM (2018d) Chronic linaclotide treatment reduces colitis-induced neuroplasticity and reverses persistent bladder dysfunction. JCI Insight 3:e121841.

Grundy L, Erickson A, Brierley SM (2019a) Visceral pain. Annu Rev Physiol 81:261-284.

Grundy L, Harrington AM, Caldwell A, Castro J, Staikopoulos V, Zagorodnyuk VP, Brookes SJ, Spencer NJ, Brierley SM (2019b) Translating peripheral bladder afferent mechanosensitivity to neuronal activation within the lumbosacral spinal cord of mice. Pain 160:793-804.

Grundy L, Caldwell A, Garcia Caraballo S, Erickson A, Schober G, Castro J, Harrington AM, Brierley SM (2020) Histamine induces peripheral and central hypersensitivity to bladder distension via the histamine H1 receptor and TRPV1. Am J Physiol Renal Physiol 318:F298-F314.

Han L, Limjunyawong N, Ru F, Li Z, Hall OJ, Steele H, Zhu Y, Wilson J, Mitzner W, Kollarik M, Undem BJ, Canning BJ, Dong X (2018) Mrgprs on vagal sensory neurons contribute to bronchoconstriction and airway hyper-responsiveness. Nat Neurosci 21:324-328.

Han L, Ma C, Liu Q, Weng HJ, Cui Y, Tang Z, Kim Y, Nie H, Qu L, Patel KN, Li Z, McNeil B, He S, Guan Y, Xiao B, Lamotte RH, Dong X (2013) A subpopulation of nociceptors specifically linked to itch. Nat Neurosci 16:174-182.

Harrington AM, Caraballo SG, Maddern JE, Grundy L, Castro J, Brierley SM (2019) Colonic afferent input and dorsal horn neuron activation differs between the thoracolumbar and lumbosacral spinal cord. Am J Physiol Gastrointest Liver Physiol 317:G285-G303.

Homma Y, Akiyama Y, Tomoe H, Furuta A, Ueda T, Maeda D, Lin AT, Kuo HC, Lee MH, Oh SJ, Kim JC, Lee KS (2020) Clinical guidelines for interstitial cystitis/bladder pain syndrome. Int J Urol 27:578-589.

Inserra MC, Israel MR, Caldwell A, Castro J, Deuis JR, Harrington AM, Keramidas A, Garcia-Caraballo S, Maddern J, Erickson A, Grundy L, Rychkov GY, Zimmermann K, Lewis RJ, Brierley SM, Vetter I (2017) Multiple sodium channel isoforms mediate the pathological effects of Pacific ciguatoxin-1. Sci Rep 7:42810.

Jiang GY, Dai MH, Huang K, Chai GD, Chen JY, Chen L, Lang B, Wang QX, St Clair D, McCaig C, Ding YQ, Zhang L (2015) Neurochemical characterization of pERK-expressing spinal neurons in histamine-induced itch. Sci Rep 5:12787.

Konthapakdee N, Grundy L, O’Donnell T, Garcia-Caraballo S, Brierley SM, Grundy D, Daly DM (2019) Serotonin exerts a direct modulatory role on bladder afferent firing in mice. J Physiol 597:5247-5264.

Kreis ME, Haupt W, Kirkup AJ, Grundy D (1998) Histamine sensitivity of mesenteric afferent nerves in the rat jejunum. Am J Physiol 275:G675G680.
La JH, Schwartz ES, Gebhart GF (2011) Differences in the expression of transient receptor potential channel V1, transient receptor potential channel $\mathrm{A} 1$ and mechanosensitive two pore-domain $\mathrm{K}^{+}$channels between the lumbar splanchnic and pelvic nerve innervations of mouse urinary bladder and colon. Neuroscience 186:179-187.

LaMotte RH, Dong X, Ringkamp M (2014) Sensory neurons and circuits mediating itch. Nat Rev Neurosci 15:19-31.

Lee JS, Han JS, Lee K, Bang J, Lee H (2016) The peripheral and central mechanisms underlying itch. BMB Rep 49:474-487.

Lee MG, Dong X, Liu Q, Patel KN, Choi OH, Vonakis B, Undem BJ (2008) Agonists of the MAS-related gene (Mrgs) orphan receptors as novel mediators of mast cell-sensory nerve interactions. J Immunol 180:22512255.

Lein ES, Hawrylycz MJ, Ao N, Ayres M, Bensinger A, Bernard A, Boe AF, Boguski MS, Brockway KS, Byrnes EJ, Chen L, Chen L, Chen TM, Chin MC, Chong J, Crook BE, Czaplinska A, Dang CN, Datta S, Dee NR, et al. (2007) Genome-wide atlas of gene expression in the adult mouse brain. Nature 445:168-176.

Lembo PM, Grazzini E, Groblewski T, O’Donnell D, Roy MO, Zhang J, Hoffert C, Cao J, Schmidt R, Pelletier M, Labarre M, Gosselin M, Fortin Y, Banville D, Shen SH, Strom P, Payza K, Dray A, Walker P, Ahmad S (2002) Proenkephalin A gene products activate a new family of sensory neuron-specific GPCRs. Nat Neurosci 5:201-209.

Liu Q, Tang Z, Surdenikova L, Kim S, Patel KN, Kim A, Ru F, Guan Y, Weng HJ, Geng Y, Undem BJ, Kollarik M, Chen ZF, Anderson DJ, Dong X (2009) Sensory neuron-specific GPCR Mrgprs are itch receptors mediating chloroquine-induced pruritus. Cell 139:1353-1365.

Liu Q, Sikand P, Ma C, Tang Z, Han L, Li Z, Sun S, LaMotte RH, Dong X (2012) Mechanisms of itch evoked by beta-alanine. J Neurosci 32:1453214537.

Liu T, Ji RR (2013) New insights into the mechanisms of itch: are pain and itch controlled by distinct mechanisms? Pflugers Arch 465:1671-1685.

Meerschaert KA, Adelman PC, Friedman RL, Albers KM, Koerber HR, Davis BM (2020) Unique molecular characteristics of visceral afferents arising from different levels of the neuraxis: location of afferent somata predicts function and stimulus detection modalities. J Neurosci 40:7216-7228.

Meixiong J, Dong X (2017) Mas-related G protein-coupled receptors and the biology of itch sensation. Annu Rev Genet 51:103-121.

Montague-Cardoso K, Malcangio M (2020) Cathepsin S as a potential therapeutic target for chronic pain. Med Drug Discov 7:100047.

Osteen JD, Herzig V, Gilchrist J, Emrick JJ, Zhang C, Wang X, Castro J, Garcia-Caraballo S, Grundy L, Rychkov GY, Weyer AD, Dekan Z, Undheim EA, Alewood P, Stucky CL, Brierley SM, Basbaum AI, Bosmans F, King GF, Julius D (2016) Selective spider toxins reveal a role for the Nav1.1 channel in mechanical pain. Nature 534:494-499.

Prato V, Taberner FJ, Hockley JR, Callejo G, Arcourt A, Tazir B, Hammer L, Schad P, Heppenstall PA, Smith ES, Lechner SG (2017) Functional and molecular characterization of mechanoinsensitive "silent" nociceptors. Cell Rep 21:3102-3115.

Reddy VB, Sun S, Azimi E, Elmariah SB, Dong X, Lerner EA (2015) Redefining the concept of protease-activated receptors: cathepsin $S$ evokes itch via activation of Mrgprs. Nat Commun 6:7864.

Rong W, Spyer KM, Burnstock G (2002) Activation and sensitisation of low and high threshold afferent fibres mediated by P2X receptors in the mouse urinary bladder. J Physiol 541:591-600.

Schmelz M (2015) Neurophysiology and itch pathways. Handb Exp Pharmacol 226:39-55.

Shim WS, Oh U (2008) Histamine-induced itch and its relationship with pain. Mol Pain 4:29.

Shinohara T, Harada M, Ogi K, Maruyama M, Fujii R, Tanaka H, Fukusumi S, Komatsu H, Hosoya M, Noguchi Y, Watanabe T, Moriya T, Itoh Y, Hinuma $S$ (2004) Identification of a $G$ protein-coupled receptor specifically responsive to beta-alanine. J Biol Chem 279:23559-23564.

Sikand P, Dong X, LaMotte RH (2011) BAM8-22 peptide produces itch and nociceptive sensations in humans independent of histamine release. J Neurosci 31:7563-7567.

Slominski AT, Zmijewski MA, Zbytek B, Brozyna AA, Granese J, Pisarchik A, Szczesniewski A, Tobin DJ (2011) Regulated proenkephalin expression in human skin and cultured skin cells. J Invest Dermatol 131:613-622.

Spencer NJ, Greenheigh S, Kyloh M, Hibberd TJ, Sharma H, Grundy L, Brierley SM, Harrington AM, Beckett EA, Brookes SJ, Zagorodnyuk VP 
(2018) Identifying unique subtypes of spinal afferent nerve endings within the urinary bladder of mice. J Comp Neurol 526:707-720.

Steinhoff M, Schmelz M, Szabo IL, Oaklander AL (2018) Clinical presentation, management, and pathophysiology of neuropathic itch. Lancet Neurol 17:709-720.

Todd AJ (2010) Neuronal circuitry for pain processing in the dorsal horn. Nat Rev Neurosci 11:823-836.

Tominaga M, Takamori K (2014) Sensitization of itch signaling: itch sensitization-nerve growth factor, semaphorins. In: Itch: mechanisms and treatment (Carstens E, Akiyama T, eds). Boca Raton, FL: CRC/Taylor and Francis.

Tseng PY, Zheng Q, Li Z, Dong X (2019) MrgprX1 mediates neuronal excitability and itch through tetrodotoxin-resistant sodium channels. itch 4 : e28.

Usoskin D, Furlan A, Islam S, Abdo H, Lonnerberg P, Lou D, HjerlingLeffler J, Haeggstrom J, Kharchenko O, Kharchenko PV, Linnarsson S, Ernfors P (2015) Unbiased classification of sensory neuron types by large-scale single-cell RNA sequencing. Nat Neurosci 18:145-153.

Van Remoortel S, Ceuleers H, Arora R, Van Nassauw L, De Man JG, Buckinx R, De Winter BY, Timmermans JP (2019) Mas-related G protein-coupled receptor C11 (Mrgprc11) induces visceral hypersensitivity in the mouse colon: a novel target in gut nociception? Neurogastroenterol Motil 31:e13623.

Wilson SR, Gerhold KA, Bifolck-Fisher A, Liu Q, Patel KN, Dong X, Bautista DM (2011) TRPA1 is required for histamine-independent, Mas-related G protein-coupled receptor-mediated itch. Nat Neurosci 14:595-602.
Wouters MM, Balemans D, Van Wanrooy S, Dooley J, Cibert-Goton V, Alpizar YA, Valdez-Morales EE, Nasser Y, Van Veldhoven PP, Vanbrabant W, Van der Merwe S, Mols R, Ghesquière B, Cirillo C, Kortekaas I, Carmeliet P, Peetermans WE, Vermeire S, Rutgeerts P, Augustijns P, et al. (2016) Histamine receptor H1-mediated sensitization of TRPV1 mediates visceral hypersensitivity and symptoms in patients with irritable bowel syndrome. Gastroenterology 150:875-887.e879.

Xu L, Gebhart GF (2008) Characterization of mouse lumbar splanchnic and pelvic nerve urinary bladder mechanosensory afferents. J Neurophysiol 99:244-253.

Yoshimura N, Ogawa T, Miyazato M, Kitta T, Furuta A, Chancellor MB, Tyagi P (2014) Neural mechanisms underlying lower urinary tract dysfunction. Korean J Urol 55:81-90.

Zagorodnyuk VP, Costa M, Brookes SJ (2006) Major classes of sensory neurons to the urinary bladder. Auton Neurosci 126:390-397.

Zagorodnyuk VP, Gibbins IL, Costa M, Brookes SJ, Gregory SJ (2007) Properties of the major classes of mechanoreceptors in the guinea pig bladder. J Physiol 585:147-163.

Zhang Y, Li S, Yecies T, Morgan T, Cai H, Pace N, Shen B, Wang J, Roppolo JR, de Groat WC, Tai C (2019) Sympathetic afferents in the hypogastric nerve facilitate nociceptive bladder activity in cats. Am J Physiol Renal Physiol 316:F703-F711.

Zhu Y, Hanson CE, Liu Q, Han L (2017) Mrgprs activation is required for chronic itch conditions in mice. Itch (Phila) 2:e9.

Zylka MJ, Dong X, Southwell AL, Anderson DJ (2003) Atypical expansion in mice of the sensory neuron-specific Mrg G protein-coupled receptor family. Proc Natl Acad Sci USA 100:10043-10048. 